Article

\title{
Bloch Oscillations in the Chains of Artificial Atoms Dressed with Photons
}

\author{
Ilay Levie and Gregory Slepyan * \\ School of Electrical Engineering, Tel Aviv University, Tel Aviv 39040, Israel; ilaylevie@gmail.com \\ * Correspondence: slepyan@post.tau.ac.il; Tel.: +972-54-737-89-17
}

Received: 28 April 2018; Accepted: 2 June 2018; Published: 6 June 2018

\begin{abstract}
We present a model of one-dimensional chain of two-level artificial atoms driven with DC field and quantum light simultaneously in a strong coupling regime. The interaction of atoms with light leads to electron-photon entanglement (dressing of the atoms with light). The driving via dc field leads to the Bloch oscillations (BO) in the chain of dressed atoms. We consider the mutual influence of dressing and $\mathrm{BO}$ and show that scenario of oscillations dramatically differs from predicted by the Jaynes-Cummings and Bloch-Zener models. We study the evolution of the population inversion, tunneling current, photon probability distribution, mean number of photons, and photon number variance, and show the influence of $\mathrm{BO}$ on the quantum-statistical characteristics of light. For example, the collapse-revivals picture and vacuum Rabi-oscillations are strongly modulated with Bloch frequency. As a result, quantum properties of light and degree of electron-photon entanglement become controllable via adiabatic dc field turning. On the other hand, the low-frequency tunneling current depends on the quantum light statistics (in particular, for coherent initial state it is modulated accordingly the collapse-revivals picture). The developed model is universal with respect to the physical origin of artificial atom and frequency range of atom-light interaction. The model is adapted to the 2D-heterostructures ( $\mathrm{THz}$ frequencies), semiconductor quantum dots (optical range), and Josephson junctions (microwaves). The data for numerical simulations are taken from recently published experiments. The obtained results open a new way in quantum state engineering and nano-photonic spectroscopy.
\end{abstract}

Keywords: Bloch oscillations; Rabi-waves; electron-photon entanglement; artificial atoms

\section{Introduction}

The early quantum theory of electrical conductivity in crystal lattices by Bloch, Zener and Wannier [1-4] led to the prediction that a homogeneous dc field induces an oscillatory rather than uniform motion of the electrons. These so-called Bloch oscillations (BO) have been observed in bulk N-doped GaAs at a lattice temperature of $300 \mathrm{~K}$ in high fields up to $300 \mathrm{kV} / \mathrm{cm}$ [5], as well as different types of artificial systems such as semiconductor superlattices [6], interacting atoms in optical lattices [7,8], ultracold atoms [9-14], light intensity oscillations in waveguide arrays [15-21], acoustic waves in layered and elastic structures [22], and atomic oscillations in Bose-Einstein condensates [23] among others. Several recent studies have investigated the dynamics of cold atoms in optical lattices subject to ac forcing; the theoretically predicted renormalization of the tunneling amplitudes has been verified experimentally. The recent observations include global motion of the atom cloud, such as giant "super-Bloch oscillations" [24]. As a result, BO transformed from the specific contra intuitive model to the general experimentally-supported physical concept of oscillatory motion of wave packets placed in a periodic potential when driven by a constant force $[8,25]$.

Rabi oscillations (RO) are periodical transitions of a two-level quantum system between its stationary states under the action of an AC driving field [26,27]. The phenomenon was theoretically 
predicted by Rabi for the nuclear spins in radio-frequency magnetic field [28] and afterwards, discovered in various physical systems, such as electromagnetically driven individual atoms [29] including the case of Rydberg atomic states [30], semiconductor quantum dots (QDs) [31] and different types of solid-state qubits (superconducting charge qubits based on Josephson junctions [32-34], spin qubits [35], semiconductor charge qubits [36]). The textbook picture of the Rabi effect is given by the Jaynes-Cummings model [26,27]. It implied that the concept of the dressed atom with light was correspondent to the quantum entanglement of electrons and photons. This model can be essentially modified by a set of additional features, such as the broken inversion symmetry [37], the propagation of RO over the chains of coupled atoms in the form of special waves (Rabi-waves), and depolarization due to the local fields [38-43]. It opens the possibility of the generation of a variety of entangled quantum states, which could have a great impact on the search for universal and efficient quantum computation processes [44], the electrically tunable optical nano-antennas with highly directive emission [39,40,43], quantum sensing [45], and metrology [46]. Recent theoretical progress in this area is associated, in particular, with the novelty of inter-atomic coupling mechanisms $[47,48]$, which manifests themselves in surprising thermodynamic behavior of the specific waveguiding arrays, which recently was experimentally verified [49].

In this paper, we build a theoretical model of a chain of coupled two-level quantum elements exposed to quantum light and driven via bias voltage. We consider the case of strong coupling of light with charge carriers, which lead to the entanglement of electron-photon quantum states. This model describes BO of electrons dressed with light and their mutual influence with RO. Our model has a significant degree of generality: It relates to the systems of different physical origin and various frequency ranges. We consider its application to semiconductor heterostructures $(\mathrm{THz}$ frequencies), semiconductor quantum dots (visible frequencies), and Josephson junctions (microwaves). For brevity, we refer to every of these artificial two-level quantum objects as an "atom" regardless of its physical implementation.

We develop the model taking into account conditions of real experiments. For example, the coherent inter-sub-band excitation of heterostructure in $\mathrm{THz}$ region has been done by the ultrashort (femtosecond) pulses [50]. On this account, we generalized our model for the case of electromagnetic pulse, advancing the secondary quantization of fields to the case of pulses. Our model is based on some conventional simplifications. We use rotating-wave approximation (RWA) and neglect any damping. This requires the fulfillment of certain relations between the frequencies (transmission frequency, light frequency, Rabi frequency, and Bloch frequency) and between some characteristic times (coherence time and attenuation time). Our calculations have been made for real physical parameters of atoms and their environment, and accessibility of these relations have been supported by the achievements of modern technologies and the data of published experiments. It allows the design of installations for potential future experiments in this branch.

The classical analog of our investigation is Rabi-Bloch oscillations (RBO) predicted in [51,52]. The quantum origin of light makes the subject dramatically changing. The classical light in the RBO case does not undergo a reverse reaction from electrons due to their transitions from the lower level to upper one and vice versa. Therefore, the light plays role of the effective refractive medium for the electron wavepacket, which guides the spatial propagation of the Rabi-wave [38-41]. For the case of quantum light, the quantum electron transitions are accompanied by time-by-time emission-absorption of photons. Therefore, it is impossible to consider the individual electrons in the capacity of consistent Bloch oscillators. The main aim of the present paper is the analysis of mutual influence of RO and BO for the case of quantum light based on the fundamental principles of quantum optics [26]. Exactly, electrons dressed with photons compose the type of quasi-particles, which are helpful for description of the considered complicated dynamics, using the picturesque BO language. The main novel predictions are: (i) The electron-photon entanglement is modulated with Bloch frequency via $\mathrm{BO}$ in dc field; and (ii) the motion of quasiparticle as a comprehensive whole is modulated with Rabi frequency via 
inter-band optical transitions. We hope that these basic results will be able to stimulate the statement of new experiments.

The paper is organized as follows. In Section 2, we review the model and basic assumptions, formulate Hamiltonian at the Wannier-Fock basis and obtain equations of motion for probability amplitudes. In Section 3, we obtain the approximate analytical solution of equations of motion basing on quasi-classical concept. In Section 4, we describe and discuss the results of numerical calculations for electron Gaussian wave packets and different initial states of light (coherent state, vacuum field, double-Fock state entangled with Gaussian wave packet). In Section 5, we analyze the potential implementations of future experiments. The main results of the work and some promising tasks for future activity are formulated in Section 6.

\section{Statement of the Problem and Calculation Technique}

\subsection{Physical System and Model}

Let us consider a one-dimensional (1D) structure of identical atoms placed over a line with period $a$ (see for example Figure 1a). Some other types of artificial atom chains are shown in Figures 2 and 3. Each atom is considered as a two-level Fermion system with transition frequency $\omega_{0}$. The location of the atoms in the lattice points is determined by the radius-vector $\mathbf{R}_{p}=\mathbf{e} p a, p=0,1, \ldots, N$, where $N$ is a number of atoms. We assume the tunneling to be the predominant mechanism of interatomic coupling and neglect other ones (such as Förster and the radiation field transfer). As it was shown in [41], such an assumption can be justified for a wide range of realistic parameter values.

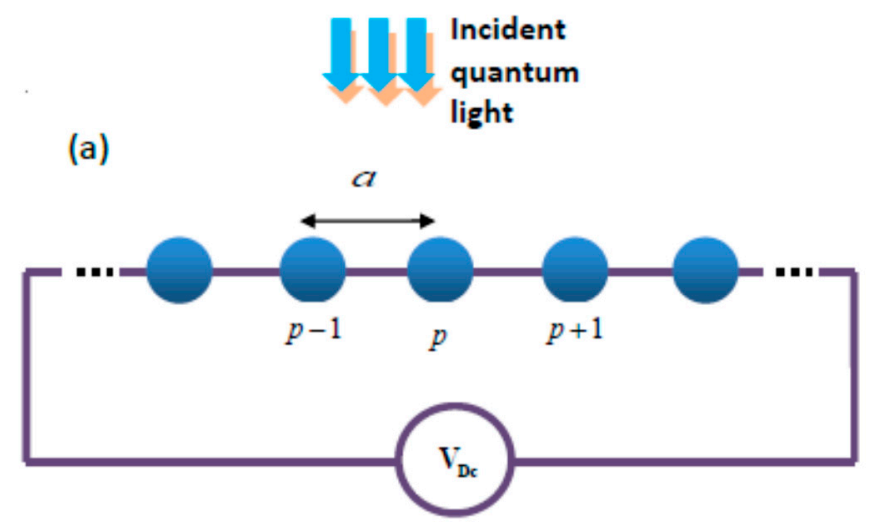

(b)

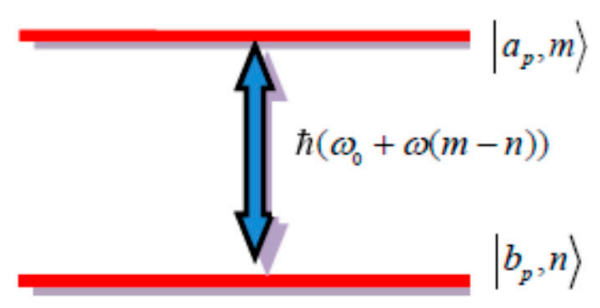

Figure 1. (a) General illustration of the periodic two-level atomic chain used as a model indicating $\mathrm{BO}$ of electrons dressed with optical photons. It is excited with incident quantum light in the strong coupling regime. It is driven with dc voltage applied to the ends. The neighboring atoms are coupled via interatomic tunneling with different values of penetration for the ground and excited states. (b) Ground and excited energy levels of single two-level atom, separated by the transition energy, $\hbar \omega_{0}$. The transition from the ground level to excited one (and vice versa) is accompanied with absorption (emission) of a single photon with energy, $\hbar \omega$. 
Before starting the consideration, we will discuss the potential degree of correspondence of our model to the different types of chains. Three rather conventional simplifications have been done: (i) We neglect all types of damping; (ii) we use so called rotating-wave approximation (RWA) [26]; and (iii) we assume the atomic chain to be infinitely long and perfectly periodic.

Assumption (i) relates to the case for scattering and radiation times strongly exceed the Bloch and Rabi periods. Because of RO for the quantum light are non-periodic, we imply here the value $t_{R} \cong \Omega_{\langle n\rangle}^{-1}$, as the Rabi-period ( $\langle n\rangle$ is the mean number of photons). These conditions are met with a large margin in superconductor junctions (artificial fluxonium atoms) [53-56]. The damping of BO and $\mathrm{RO}$ in heterostructures is defined by the electron scattering and decoherence, respectively. The recent progress in molecular beam epitaxy allowed achieving the values of scattering time $\tau \cong 10^{-10} \mathrm{~s}$ in the ultra-high-quality AlGaAs/GaAs heterostructures [57]. For this case $\mathrm{BO}$ may be considered as ballistic, while dephasing becomes the dominant component of the damping for coherent inter-sub-band $\mathrm{THz}$ transitions (the typical values of characteristic times are 100-300 fs [50]). Such values are comparable with typical Bloch and Rabi periods; therefore, the damping does not manifest itself. In [50], the authors experimentally observed the manifestation of RO for the pulse with duration $200 \mathrm{fs}$, which does not exceed the dephasing time and is comparable with Rabi period (1-2 RO cycles over the pulse). For validity of our model to this case, we will consider interaction with rather short pulse, of which the duration, however, strongly exceeds the period of high-frequency filling and guaranties RWA validity.

The perfect periodicity means the identity and a rather large number of atoms in the chain. Its implementation is rather simple for 2D-heterostructures (for example, a chain of 51 element was used in experiments [50]). The problem is not so easy for QD-chains because of QDs comprise hundreds or thousands of real atoms, with inevitable variations in size and shape and, consequently, unavoidable variability in their energies and relaxation times $[58,59]$. One of the most promising technologies for applications in quantum photonics is the embedding of QDs within nanowires [59]. The QDs form at the apex of a GaAs/AlGaAs interface, are highly stable, and can be positioned with nanometer precision relative to the nanowire centre. As it was found [59], there is a chain of bright, nanoscale emitters in the red. QD-in-nanowire mimics very closely a two-level atom with high associated lifetime ( $\cong 450 \mathrm{ps}$ ). One more way is using a scanning tunneling microscope to create QDs with identical, deterministic sizes [58]. As it was mentioned in [58], the reached digital fidelity opens the door to QD architectures free of intrinsic broadening. This makes it reasonable to recommend our model of 1D QD-chain for visible light applications.

There exist different ways to theoretically describe complex quantum systems strongly interacted with quantum light. In quantum optics an "all-matter" picture is widely used, where the dynamics of light is integrated out (for example, in the optical Bloch equations [60,61]). In Refs. [62,63] the light-matter interaction is treated in an "all-light" picture (Lippmann-Schwinger equation approach). We use as the theoretical approach the probability amplitude method, generalized for the case of 1D chains driven via homogeneous dc field. It consists of solving of the Schrödinger equation for wave function, which is the superposition of various Wannier-Fock states for atom-light system.

To solve equations of motion for probability amplitudes analytically, we use the quasi-classical concept. The dressed electron is described as a wavepacket prepared with a well-defined quasi-momentum. The motion of quasiparticle center of mass is governed by the Newton's law, while the internal degrees of freedom have been described by means of quantum theory, based on the concept of electron-photon entanglement widely used in quantum optics [26,27]. Thus, the position of the quasi-particle center periodically evolves with a Bloch frequency corresponding to the quasi-momentum scanning a complete Brillouin zone. Such an approach was used for BO of conductive electrons in $[8,64]$ and will be adopted for the case of dressed electrons in this paper.

In the general case, the equations of motion were integrated numerically with simplification through RWA. However, the use of the RWA may not describe the atom-light interaction when the coupling becomes sufficiently strong (ultrastrong coupling) [65]. Both lower and upper fundamental 
limitations of ultrastrong coupling of atoms and light were recently formulated in [66]. We bounded our consideration by the strong coupling regime assuming the lower limitation in [66] not to be reached.

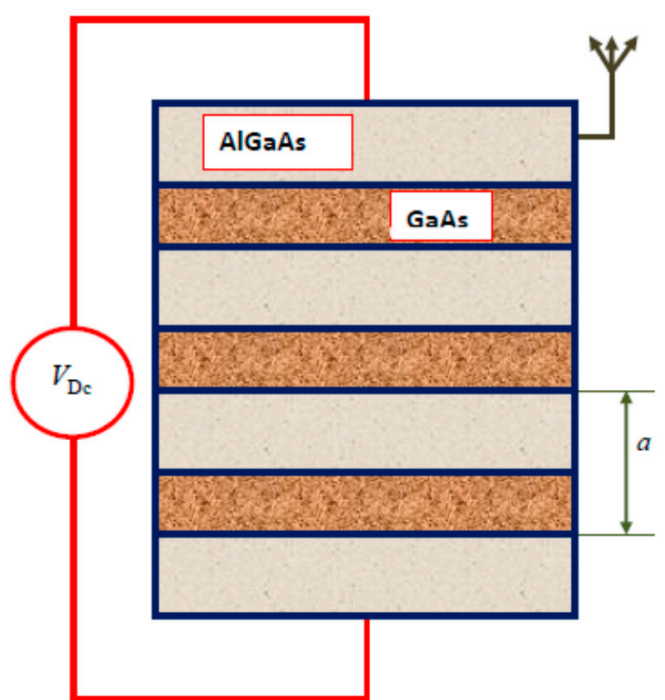

(a)
THz-

nanoantenna

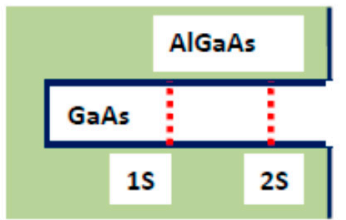

(b)

Figure 2. Schematic diagram of 2D semiconductor heterostructure. (a) n-type modulation-doped quantum well sample consisting from GaAs wells considered as artificial atoms forming a chain. The atoms are separated from each other by AlGaAs barriers. The coupling is governed by the inter-barrier electron tunneling. The dc voltage is applied to the ends of the system. The femtosecond $\mathrm{THz}$ pulse comes in through nanoantenna. (b) $1 \mathrm{~S} \Leftrightarrow 2 \mathrm{~S}$ inter-sub-band transition, which is coherently excited by the pulse with narrow spectrum (central frequency is resonant with quantum transition).

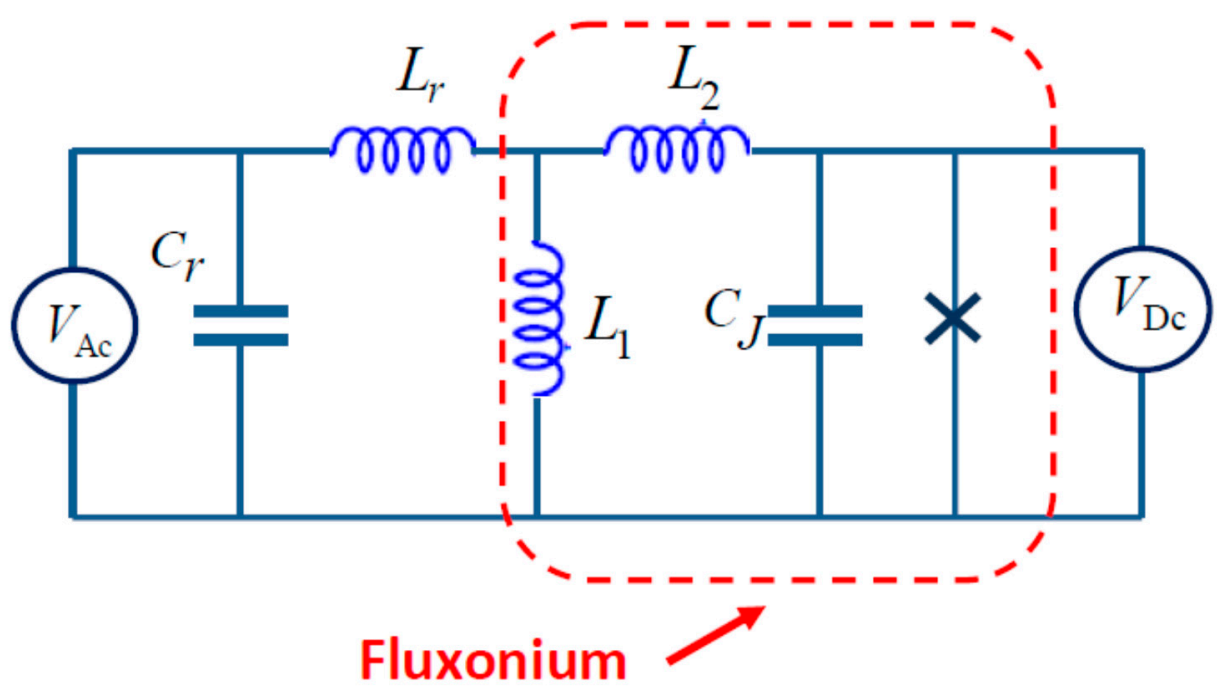

Figure 3. Circuit diagram of a Josephson junction (crossed), which is inductively coupled with microwave resonator. The inductance $L_{2}$ describes the inductive environment of the junction. The inductance $L_{1}$ is a tool of control of the "junction-resonator" coupling (it is assumed $L_{2}>>L_{1}$ ). Josephson energy $E_{J}$ strongly exceeds charging energy $E_{C}\left(E_{J}>>E_{C}\right)$. We model the resonator as an LC-circuit with effective inductance $L_{r}$ and effective capacitance $C_{r}$. The single fluxonium plays role of atomic chain due to the interaction with inductive environment [53-56] (BO take place in the space of quasi-charge). 


\subsection{Hamiltonian in Wannier-Fock Basis}

Let us denote with $\left|a_{p}\right\rangle$ and $\left|b_{p}\right\rangle$, the Wannier wave functions centered at $p$-th atom in the excited and ground states, respectively (Figure 1). The two neighboring atoms are coupled via the electron tunneling, such that only intra-band tunnel transitions are permitted. It means that the electron due to the tunneling can go from state $\left|a_{p}\right\rangle$ to state $\left|a_{p+1}\right\rangle$ and from state $\left|b_{p}\right\rangle$ to state $\left|b_{p+1}\right\rangle$ only. The transitions between ground and excited states of different atoms are forbidden: $\left\langle a_{p \pm 1} \mid b_{p}\right\rangle \approx 0$.

Let the chain be exposed to a single-mode quantum light, which electric field operator is $\hat{\mathbf{E}}=\hat{\mathbf{E}}^{(+)}+\hat{\mathbf{E}}^{(-)}=\mathbf{e} \sqrt{\hbar \omega / 2 \varepsilon_{0} V}\left(\hat{a}+\hat{a}^{+}\right)$, where $V$ is the normalizing volume, $\mathbf{e}$ is the unit polarization vector, $\hat{\mathbf{E}}^{(+)}=\mathbf{e}\left(\hbar \omega / 2 \varepsilon_{0} V\right)^{1 / 2} \hat{a}, \hat{\mathbf{E}}^{(-)}=\mathbf{e}\left(\hbar \omega / 2 \varepsilon_{0} V\right)^{1 / 2} \hat{a}^{+}$are positive and negative frequency components of field operator, $\hat{a}, \hat{a}^{+}$are creation-annihilation operators, respectively (an $\exp (-i \omega t)$ time dependence of the light is implicit). The field is assumed to be homogeneous over the chain axis. Such assumption corresponds to the chain excited by the normally incident wide laser beam, or specially-symmetric eigen-mode of microcavity, photonic crystal; etc. The chain is driven by an electrostatic (dc) field directed along the axis with responsibility for BO. We will consider the case of dipole interaction in the regime of strong coupling and assume the resonant condition $\omega_{0} \approx \omega$ to be fulfilled. The system under consideration exhibits complex single-particle BO of the electrons entangled with photons, for which the theoretical framework will be introduced.

The system is described by the total Hamiltonian (see Appendix A):

$$
\hat{H}=\hat{H}_{0}+\hat{H}_{p h}+\hat{H}_{T}+\hat{H}_{I, p h}+\hat{H}_{I, d c} .
$$

Here, the first term represents the free motion of the chain with absence of both light and dc field. It is given by $\hat{H}_{0}=\left(\hbar \omega_{0} / 2\right) \sum_{p} \hat{\sigma}_{z p}$, where $\hat{\sigma}_{z p}=\left|a_{p}\right\rangle\left\langle a_{p}|-| b_{p}\right\rangle\left\langle b_{p}\right|$. The second term $\hat{H}_{p h}=\hbar \omega \hat{a}^{+} \hat{a}$ is the Hamiltonian of free electromagnetic field. The term

$$
\begin{aligned}
\hat{H}_{T}= & -t_{a} \sum_{p}\left(\left|a_{p}\right\rangle\left\langle a_{p+1}|+| a_{p}\right\rangle\left\langle a_{p-1}\right|\right)- \\
& -t_{b} \sum_{p}\left(\left|b_{p}\right\rangle\left\langle b_{p+1}|+| b_{p}\right\rangle\left\langle b_{p-1}\right|\right)
\end{aligned}
$$

is Hamiltonian of electron tunneling, $t_{a, b}$ are the penetration energies of potential barrier at the excited and ground states, respectively.

The component

$$
\hat{H}_{I, p h}=\hbar g \sum_{p} \hat{\sigma}_{p}^{+} \hat{a}+\text { H.c. }
$$

describes the atom-light interaction, where $g=-\sqrt{\omega / 2 \hbar \varepsilon_{0} V}\left(\mathbf{e} \cdot \mathbf{d}_{a b}\right)$ is the interaction constant, $\mathbf{d}_{a b}$ is the dipole moment, H.c. means Hermitian conjugation. The transition dipole moments in the chain is assumed to be vectors of identical values and orientations. The operators $\hat{\sigma}_{p}^{+}=\left|a_{p}\right\rangle\left\langle b_{p}\right|$, $\hat{\sigma}_{p}^{-}=\left|b_{p}\right\rangle\left\langle a_{p}\right|$ are creation-annihilation operators of excited state in the $p$-th atom. The Hamiltonian (3) is written in the RWA form [26].

The last term,

$$
\hat{H}_{I, d c}=e \sum_{p}\left(\mathbf{E}_{d c} \cdot \mathbf{R}_{p}\right)\left|a_{p}\right\rangle\left\langle a_{p}\left|+e \sum_{p}\left(\mathbf{E}_{d c} \cdot \mathbf{R}_{p}\right)\right| b_{p}\right\rangle\left\langle b_{p}\right|,
$$

describes the driving via dc field $\mathbf{E}_{d c}$ and is responsible for $\mathrm{BO}$. 


\subsection{Equations of Motion}

The evolution of the system in the interaction picture is described by the Schrödinger equation $i \hbar \partial_{t}|\Psi\rangle=\hat{V}|\Psi\rangle$ with the interaction Hamiltonian given by:

$$
\hat{V}=\exp \left(\frac{i}{\hbar} \hat{H}_{p h} t\right)\left(\hat{H}_{0}+\hat{H}_{I, p h}+\hat{H}_{I, d c}+\hat{H}_{T}\right) \exp \left(-\frac{i}{\hbar} \hat{H}_{p h} t\right) .
$$

The state vector of the "atomic chain + light" system is:

$$
|\psi(t)\rangle=\sum_{n} \sum_{p}\left\{a_{p, n}(t)\left|a_{p}, n\right\rangle+b_{p, n}(t)\left|b_{p}, n\right\rangle\right\} .
$$

Here, $\left|b_{p}, n\right\rangle=\left|b_{p}\right\rangle \otimes|n\rangle,\left|a_{p}, n\right\rangle=\left|a_{p}\right\rangle \otimes|n\rangle$, are Fock states with $n$ photons and Wannier states centered around $p$-th atom in ground and excited state, respectively, $a_{p, n}$ and $b_{p, n}$ are the unknown probability amplitudes. From the Schrodinger equation we get following equations for the probability amplitudes:

$$
\begin{gathered}
i \hbar \frac{\partial a_{p, n}}{\partial t}=\left(\delta \varepsilon-\mathbf{E}_{d c} \cdot e \mathbf{R}_{p}\right) a_{p, n}+t_{a} a_{p+1, n}+t_{a}^{*} a_{p-1, n}-g \sqrt{n+1} b_{p, n+1}, \\
i \hbar \frac{\partial b_{p, n+1}}{\partial t}=-\left(\delta \varepsilon+\mathbf{E}_{d c} \cdot e \mathbf{R}_{p}\right) b_{p, n+1}+t_{b} b_{p+1, n+1}+t_{b}^{*} b_{p-1, n+1}-g \sqrt{n+1} a_{p, n},
\end{gathered}
$$

where $\delta \varepsilon=\left(\varepsilon_{a}(0)-\varepsilon_{b}(0)-2 \omega\right) / 2$. In the limit $E_{d c}=0$ the System (7) and (8) goes to the corresponding equations obtained in [41] for Rabi-waves in QD chains.

\subsection{Studying Observable Values}

We will study the values of two types. The first ones directly characterize the spatial-temporal behavior of electronic component and therefore are averaged over photonic state distribution. They are position-dependent, thus we will address to their densities per unit cell of the chain. From among these values we introduce:

(i) inversion density, given by

$$
W(p, t)=\sum_{n}\left(\left|a_{p, n}(t)\right|^{2}-\left|b_{p, n}(t)\right|^{2}\right)
$$

and

(ii) tunneling current density (see Appendix B)

$$
\begin{aligned}
J_{\text {Tunneling, } p}(t)= & J_{\text {Tunneling, } p}^{(a)}(t)+J_{\text {Tunneling, }, p}^{(b)}(t)= \\
& -i \frac{e}{2} t_{a} \sum_{n}\left(a_{p-1, n}(t)-a_{p+1, n}(t)\right) a_{p, n}^{*}(t) \\
& -i \frac{e}{2} t_{b} \sum_{n}\left(b_{p-1, n}(t)-b_{p+1, n}(t)\right) b_{p, n}^{*}(t)+\text { c.c. }
\end{aligned}
$$

The values of the second type directly characterize the quantum-statistical properties of light and therefore are spatially averaged. From among these values we will use:

(iii) photonic number distribution

$$
\widetilde{p}(n, t)=\sum_{p}\left(\left|a_{p, n}(t)\right|^{2}+\left|b_{p, n}(t)\right|^{2}\right)
$$


(iv) mean number of photons

$$
\langle n(t)\rangle=\sum_{n} n \widetilde{p}(n, t)
$$

where $\widetilde{p}(n, t)$ is given by (11),

(v) photonic number variance

$$
\delta n(t)=\sum_{n} n^{2} \widetilde{p}(n, t)-\langle n(t)\rangle^{2},
$$

where $\langle n(t)\rangle$ is given by relation (12), and

(vi) von Neumann quantum entropy

$$
S=\sum_{j} p_{j} \ln p_{j}
$$

where $p_{j}$ are the weights of the various Fock states of the statistical light distribution [44].

\section{Approximate Analytical Solution}

\subsection{Preliminaries}

The simplest and conventional model of $\mathrm{BO}$ is based on a quasi-classical approximation. It results from Equations (7) and (8) with $g=0$. Electron-photon interaction disappears (subscript $n$ becomes unnecessary) and the equations become separated. The system reads,

$$
i \hbar \frac{\partial a_{p}}{\partial t}=-e\left(\mathbf{E}_{d c} \cdot \mathbf{R}_{p}\right) a_{p}+t_{a} a_{p+1}+t_{a}^{*} a_{p-1}
$$

and the same for $b_{p}$. (the value $\delta \varepsilon$ becomes arbitrary and is taken $\delta \varepsilon=0$ ). In the absence of a dc field $\left(\mathbf{E}_{d c}=0\right)$, the eigenstate of the system corresponds to the electrons tunneling in the periodic potential and has a form of Bloch-wave $a_{p+1}(t)=a_{p} e^{i(\varphi-v t)}$, where $\varphi$ is a phase shift per unit cell, coupled with the eigen frequency by $v=2 \hbar^{-1} t_{a} \cos \varphi$. The phase shift is coupled with the continuous quasi-momentum via relation $h=\varphi / a$, conventionally restricted to the first Brillouin zone $[-\pi / a ; \pi / a]$. Under the influence of a dc field, a given Bloch-state $\left|\Psi\left(h_{0}\right)\right\rangle=N^{-1 / 2} \sum_{p}\left|a_{p}\right\rangle e^{i\left(h_{0} p a-v\left(h_{0}\right) t\right)}$ evolves up to a phase factor into the state $|\Psi(h(t))\rangle$ with $h(t)$ variation according to

$$
\frac{d h}{d t}=-\frac{e E_{d c}}{\hbar}
$$

or $h(t)=-e \hbar^{-1} E_{d c} t+h_{0}$. Thus, this evolution is periodic with a Bloch frequency corresponding to the time required for the quasi-momentum to scan a full Brillouin zone. It is described by the substitution $\varphi(t)=h(t) a=-\omega_{B} t+\varphi_{0}$, where $\omega_{B}=-e \hbar^{-1} E_{d c}$ is the Bloch frequency, $h_{0}=\varphi_{0} a$. The obtained solution relates also to the rather wide wave-packet with well-defined quasi-momentum. The periodic motion of its geometrical center corresponds to the quasi-classical model of $\mathrm{BO}$.

The main idea of the analytical solution considered next is based on hypothesis that the total dynamics of quasiparticles described by Equations (8) and (9) are reducible to the superposition of two interacting partial motions: (i) internal motion, which is dictated by dressing; and (ii) external motion of the quasiparticle as a whole. The internal motion is of completely quantum origin and doesn't allow any classical interpretation. It corresponds to the Rabi-wave and plays the role of the tunneling in the simplest case of $\mathrm{BO}$ considered before. The external motion is a motion of geometric center and will be described quasi-classically, following Equation (16).

\subsection{Details}

We consider the partial solutions of System (7) and (8) in the absence of a dc field $\left(\mathbf{E}_{d c}=0\right)$ and the case of zero detuning $\left(\omega=\omega_{0}\right)$. We use the ansatz $a_{p+1, n}(t)=a_{p, n}(t) e^{i \varphi}, b_{p+1, n}(t)=b_{p, n}(t) e^{i \varphi}$, $a_{p, n}(t)=\widetilde{a}_{p n} e^{-i v t}, b_{p, n}(t)=\widetilde{b}_{p n} e^{-i v t}$, where $\varphi$ is a given phase shift per unit cell, $v$ is the unknown 
eigen frequency, $\widetilde{a}_{p n}, \widetilde{b}_{p n}$ are unknown constant coefficients. Making use of Equations (7) and (8) we obtain for them the matrix equation

$$
\left(\begin{array}{cc}
\hbar v-2 t_{a} \cos \varphi & -g \sqrt{n+1} \\
-g \sqrt{n+1} & \hbar v-2 t_{b} \cos \varphi
\end{array}\right) \cdot\left(\begin{array}{c}
\widetilde{a}_{p n} \\
\widetilde{b}_{p, n+1}
\end{array}\right)=0 .
$$

The eigenfrequencies are found from its characteristic equation

$$
\left(\hbar v-2 t_{a} \cos \varphi\right)\left(\hbar v-2 t_{b} \cos \varphi\right)-g^{2}(n+1)=0
$$

and given by

$$
\hbar v_{1,2}(\varphi)=\left\{\left(t_{a}+t_{b}\right) \cos \varphi \pm \sqrt{\left(t_{a}-t_{b}\right)^{2} \cos ^{2} \varphi+g_{n}^{2}}\right\}
$$

where $g_{n}=g \sqrt{n+1}$. The correspondent eigenstates are

$$
\begin{aligned}
& \left|\Psi_{n, 1}\right\rangle=\frac{e^{-i(n+1) \omega t} \cdot e^{-i v_{1} t}}{\sqrt{N\left(1+\Delta_{n}^{2}(\varphi)\right)}} \sum_{p} e^{i p \varphi}\left(\left|a_{p}, n\right\rangle-\Delta_{n}(\varphi)\left|b_{p}, n+1\right\rangle\right), \\
& \left|\Psi_{n, 2}\right\rangle=\frac{e^{-i(n+1) \omega t} \cdot e^{-i v_{2} t}}{\sqrt{N\left(1+\Delta_{n}^{2}(\varphi)\right)}} \sum_{p} e^{i p \varphi}\left(\Delta_{n}(\varphi)\left|a_{p}, n\right\rangle+\left|b_{p}, n+1\right\rangle\right),
\end{aligned}
$$

where

$$
\Delta_{n}(\varphi)=\frac{g_{n}}{\left(t_{a}-t_{b}\right) \cos \varphi+\sqrt{\left(t_{a}-t_{b}\right)^{2} \cos ^{2} \varphi+g_{n}^{2}}} .
$$

The States (14) and (15) describe the travelling of transitions between the states $\left|a_{p}, n\right\rangle$ and $\left|b_{p}, n+1\right\rangle$ along the chain (so called Rabi-waves [41]).

The periodicity of the lattice leads to a band structure of the energy spectrum of the dressed electrons in the entangled eigenstates $\left|\Psi_{\alpha, n}(h)\right\rangle$ with the corresponding eigenenergies $\hbar v_{1,2}(h, n)$. They are labeled by the discrete number of photons $n$ and the continuous quasi-momentum $h$. Under the influence of a dc field, weak enough not to induce inter-band transitions, the state $\left|\Psi_{\alpha, n}\left(h_{0}\right)\right\rangle$ evolves up to a phase factor into the state $\left|\Psi_{\alpha, n}(h(t))\right\rangle$ with $h(t)$ variation according to Equation (16). This evolution is periodic with a Bloch frequency $\omega_{B}$ corresponding to the time required for the quasi-momentum to scan a full Brillouin zone. It leads to the exchange,

$$
\Delta_{n}(\varphi) \rightarrow \Delta_{n}(t)=\frac{g_{n}}{\left(t_{a}-t_{b}\right) \cos \left(\omega_{B} t-\varphi_{0}\right)+\sqrt{\left(t_{a}-t_{b}\right)^{2} \cos ^{2}\left(\omega_{B} t-\varphi_{0}\right)+g_{n}^{2}}} .
$$

The distribution of photonic probabilities for the state $\left|\Psi_{n, 1}\right\rangle$ is given by

$$
p_{1}(m, t)= \begin{cases}\frac{1}{1+\Delta_{n}^{2}(t)}, & m=n \\ \frac{\Delta_{n}^{2}(t)}{1+\Delta_{n}^{2}(t)}, & m=n+1 \\ 0, & \text { others }\end{cases}
$$

The average number of photons is

$$
\left\langle n_{1}(t)\right\rangle \approx n+\frac{\Delta_{n}^{2}(t)}{1+\Delta_{n}^{2}(t)}
$$

where the value $\Delta_{n}(t)$ is given by Relation (23). The similar elementary calculations for the state $\left|\Psi_{n, 2}\right\rangle$ give

$$
\left\langle n_{2}(t)\right\rangle \approx n+\frac{1}{1+\Delta_{n}^{2}(t)}
$$


For photonic number variances we have

$$
\delta n_{1}=\delta n_{2}=\frac{\Delta_{n}^{2}(t)}{\left(1+\Delta_{n}^{2}(t)\right)^{2}} .
$$

Here, von Neumann entropy [44] accumulated at the given states of light is equal for both states and given by

$$
S_{n, 1}(t)=S_{n, 2}(t)=\frac{1}{1+\Delta_{n}^{2}(t)} \ln \left(\frac{1}{1+\Delta_{n}^{2}(t)}\right)+\frac{\Delta_{n}^{2}(t)}{1+\Delta_{n}^{2}(t)} \ln \left(\frac{\Delta_{n}^{2}(t)}{1+\Delta_{n}^{2}(t)}\right) .
$$

Due to $\mathrm{BO}$, characteristics of quantum light, such as the photonic probabilities, the average number of photons, and the Neiman entropy are oscillatory functions with Bloch frequency $\omega_{B}$. The amplitude of these non-monochromatic oscillations is strongly dependent on the relation between penetrations of potential barrier in the ground and excited states. In particular, as obeyed from Equation (17), for the case of identical tunneling penetrations at the ground and excited states $\left(t_{a} \rightarrow t_{b}\right)$ the value $\Delta_{n}$ becomes constant, and oscillations vanish. The oscillating effect is suppressed likewise with the increasing of photons number $n$. As a consequence, a wave packet of entangled electron-photon prepared with a well-defined quasi-momentum will also oscillate in position with amplitude $2 \hbar\left(t_{a}+t_{b}\right) / e E_{d c}$.

Finally, we note that there is an interesting ability for quantum state control via adiabatically turning the dc field on and off. Starting from $E_{d c}=0$ we proceed to turn on $E_{d c}$ until its maximal value. Thereafter we slowly turn $E_{d c}$ off and end it at the moment $t=T$. This process corresponds to the turning the phase from initial value $\varphi=\varphi_{0}$ until the final one given by

$$
\Phi=\varphi(T)=\varphi_{0}-e \hbar^{-1} a^{-1} \int_{0}^{T} E_{d c}(\tau) d \tau .
$$

The optimal choice of the turning time $T$ makes the arbitrary value of the phase turn reachable. As a result, the DC field via $\mathrm{BO}$ becomes an effective tool of control of quantum light statistics. In particular, the photonic probabilities, degree of electron-photon entanglement, and Neumann entropy may be adiabatically changed from minimal to maximal values (and vice versa) via adiabatic turning of the DC field.

\section{Numerical Modelling and Discussion}

In this section, we will show and discuss the numerical results for the different types of initial states. The System (8) and (9) was solved with the Crank-Nicolson numerical integration technique [67]. We have used periodic Born-Von Karman relations $a_{1, n}=a_{N, n}, b_{1, n}=b_{N, n}$ as boundary conditions [41]. Let us note that the concrete form of boundary conditions has no physical importance in our case because the area of oscillations is placed rather distantly from the ends of the chain. For simplicity, we will limit our consideration by the case of zero detuning $\left(\omega=\omega_{0}\right)$.

We will consider three types of initial conditions. For two of them the electron and photon subsystems are assumed to be initially non-interacting. The electronic subsystem is prepared as a coherent superposition of Gaussian wave packets:

$$
\begin{aligned}
& a_{p}(0)=a_{0} e^{-\frac{\left(p-u_{a}\right)^{2} a^{2}}{\sigma_{a}^{2}}} e^{i k_{a} p a,} \\
& b_{p}(0)=b_{0} e^{-\frac{\left(p-u_{b}\right)^{2} a^{2}}{\sigma_{b}^{2}}} e^{i k_{b} p a},
\end{aligned}
$$

where $a_{0}, b_{0}$ are arbitrary complex values that satisfy the normalization condition, $u_{a, b}, \sigma_{a, b}, k_{a, b}$ are the position of Gaussian center, effective Gaussian width, and the initial value of quasi-momentum at the ground and excited states, respectively. 
The photonic subsystem is prepared in the next types of state:

(i) Coherent initial state; It is given by Poisson distribution $p(n)=\langle n\rangle^{n / 2} e^{-\langle n\rangle / 2} / \sqrt{n !}$, where $\langle n\rangle$ is the mean number of photons. The total wavefunction at the moment $t=0$ is given by

$$
|\psi(0)\rangle=\sum_{n} \frac{\langle n\rangle^{n / 2}}{\sqrt{n !}} e^{-\frac{\langle n\rangle}{2}} \sum_{p}\left(a_{0} e^{-\frac{\left(p-u_{a}\right)^{2} a^{2}}{\sigma_{a}^{2}}} e^{i k_{a} p a}\left|a_{p}, n\right\rangle+b_{0} e^{-\frac{\left(p-u_{b}\right)^{2} a^{2}}{\sigma_{b}^{2}}} e^{i k_{b} p a}\left|b_{p}, n\right\rangle\right)
$$

(ii) Vacuum initial state; It is given by the total wave function

$$
|\psi(0)\rangle=\sum_{p}\left(a_{0} e^{-\frac{\left(p-u_{a}\right)^{2} a^{2}}{\sigma_{a}^{2}}} e^{i k_{a} p a}\left|a_{p}, 0\right\rangle+b_{0} e^{-\frac{\left(p-u_{b}\right)^{2} a^{2}}{\sigma_{b}^{2}}} e^{i k_{b} p a}\left|b_{p}, 0\right\rangle\right),
$$

where $a_{0}, b_{0}$ are the arbitrary coefficients satisfying normalization condition;

(iii) Entangled photon-electron initial state; The electron-photon entanglement prepared initially before the driving fields would be switching on. The initial condition reads

$$
\left|\psi_{n}(0)\right\rangle=C_{0} \sum_{p} e^{-\frac{(p-u)^{2} a^{2}}{\sigma^{2}}}\left(\left|a_{p}, n\right\rangle+\left|b_{p}, n+1\right\rangle\right),
$$

where $C_{0}$ is normalization constant. The value of inversion for the State (34) is equal to zero.

In Figure 4, we plotted the temporal dynamics of inversion. The case of zero dc field and coherent initial light (Figure 4a) corresponds to the well-known scenario of collapse-revivals given by the Jaynes-Cummings model for the single atom [26]. The initial spatial distribution is not disturbed over the motion via inter-atomic tunneling. For the case of dc field existence and coherent initial light (Figure $4 \mathrm{~b}$ ), the collapse-revivals picture drifts due to BO: The collapses and revivals appear at the different segments of the atomic chain. The inversion behavior dramatically changes for the vacuum state of light (Figure 4c). Here, BO leads to the separation of areas of positive and negative values of inversion. The particle in the excited state (positive inversion) exhibits the single oscillation of rather high amplitude in the course a half of Bloch circle. During the other part of Bloch circle, the particle is in rest, which means its appearance in the ground state (negative inversion). The reason for this is the weak value of transparence of potential barrier at the ground level used in the shown calculations. In contrast with single atom scenario, vacuum $\mathrm{RO}$ between maximal and minimal values of inversion occurs with Bloch frequency $\omega_{B}$ instead of Rabi frequency $\Omega_{0}=2 g$.

In Figure 5, we plotted the time dependence of the tunneling current. For the absence of light (Figure 5a), the current exhibits the periodic dynamics with Bloch frequency. This picture is dramatically changed due to the light-chain coupling both for coherent and vacuum states of light (Figure $5 b, c)$, respectively. For the coherent light, the tunnel current is modulated in exact compliance with the collapse-revivals picture. This result is rather counterintuitive; it demonstrates the influence of the quantum-light statistics on the low-frequency motion of the charges driven separately by a dc field. It makes the spectra of the tunneling current more wide and various wherewith in the case of ordinary BO. It opens a new promising avenue in the spectroscopy of nano-circuits and nano-devices based on the synthesis of quantum optical and dc tools of control [51,52]. For the vacuum state of light, the tunnel current periodically evolves through one pulse after another with frequency $\omega_{B}$ instead of continuous periodic behavior in the case of ordinary $\mathrm{BO}$ [8-25]. Such evolution is accompanied by the modulation with Rabi frequency. The results demonstrate high and various mutual interactions of $\mathrm{RO}$ and $\mathrm{BO}$. 

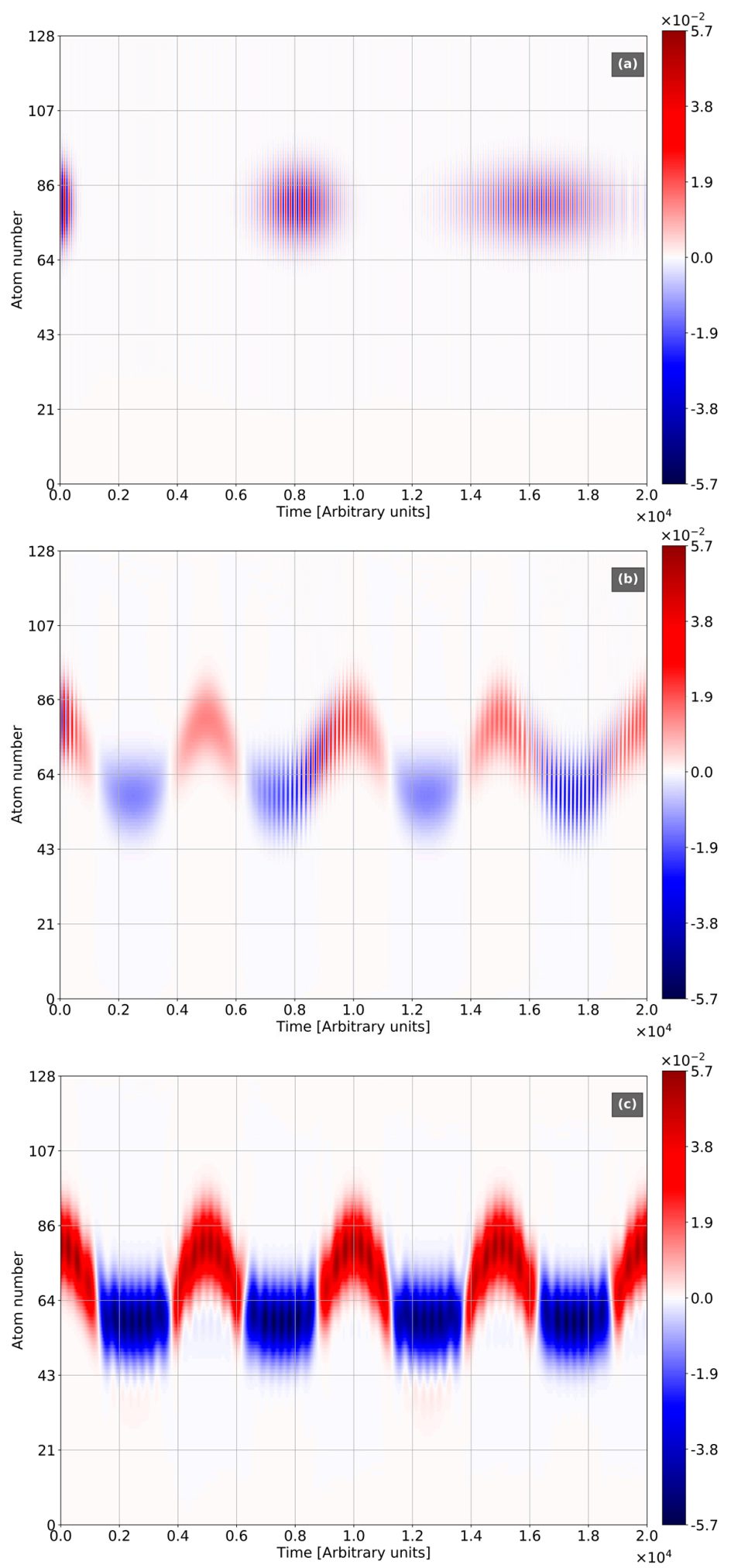

Figure 4. Space-time distribution of inversion density. All frequencies are normalized to the frequency of quantum transition $\omega_{0}$. Sampling Frequency $=4$. The number of atoms $N=128$, Rabi-frequency $\Omega_{\langle n\rangle}=0.025$, electron initial Gaussian with $a_{0}=1, b_{0}=0, u_{a}=80, \sigma_{a}=10, k_{a}=0$. (a) Light in the coherent state with $\langle n\rangle=25$. $\omega_{B}=0, t_{a} / \omega_{B}=t_{b} / \omega_{B}=10$. (b) Light in the coherent state with $<n>=25 . \omega_{B}=0.0008, t_{a} / \omega_{B}=10, t_{b} / \omega_{B}=1$. (c) light in vacuum. $\omega_{B}=0.0008, t_{a} / \omega_{B}=10$, $t_{b} / \omega_{B}=1$. 

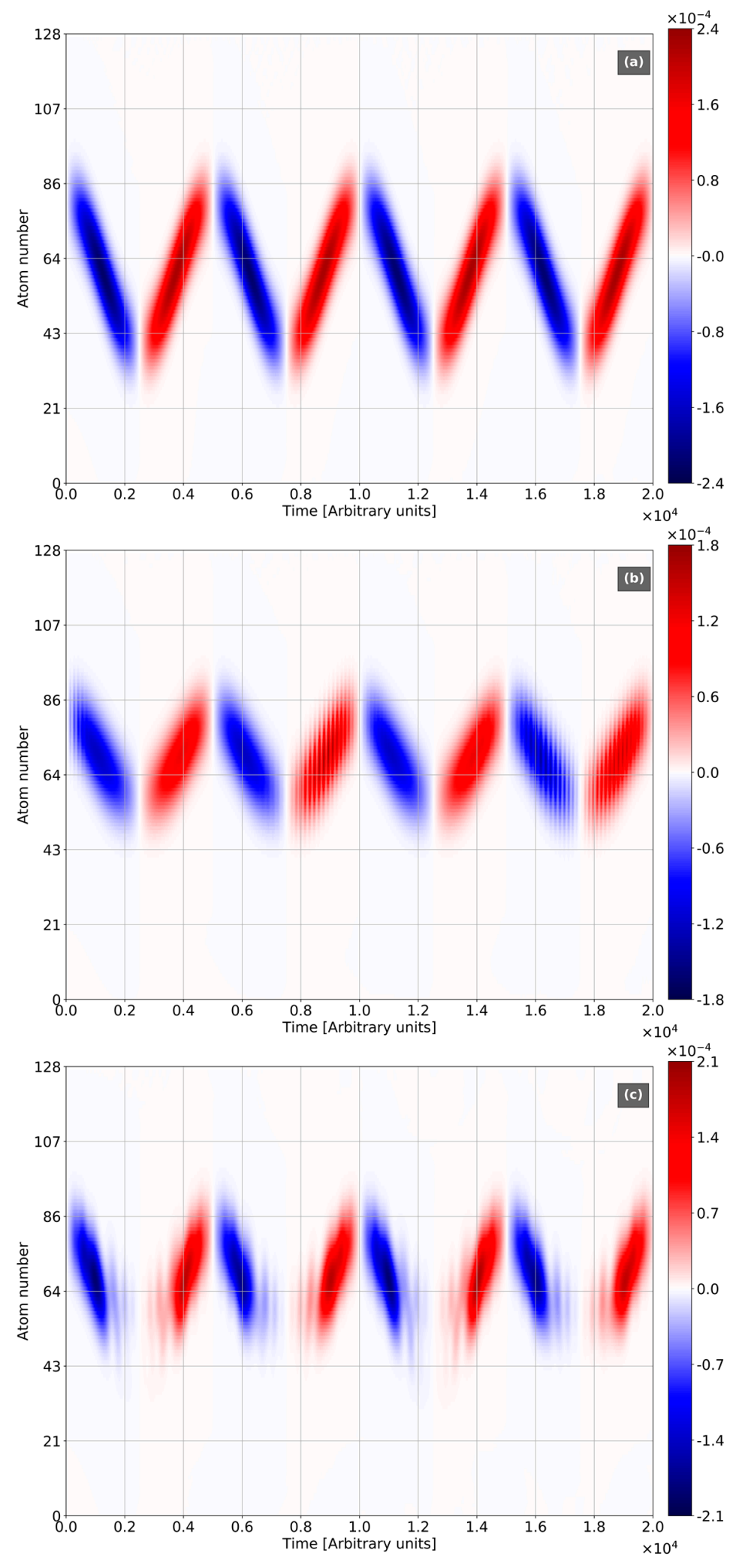

Figure 5. Space-time distribution of tunnel current density. All frequencies are normalized to the frequency of quantum transition $\omega_{0}$. Sampling Frequency $=4$. The number of atoms $N=128$, Rabi-frequency $\Omega_{\langle n\rangle}=0.025$, electron initial Gaussian with $a_{0}=1, b_{0}=0, u_{a}=80, \sigma_{a}=10, k_{a}=0$. (a) Light in the coherent state with $\langle n\rangle=25 . \omega_{B}=0, t_{a} / \omega_{B}=t_{b} / \omega_{B}=10$. (b) Light in the coherent state with $\langle n\rangle=25$. $\omega_{B}=0.0008, t_{a} / \omega_{B}=10, t_{b} / \omega_{B}=1$. (c) Light in vacuum state. $\omega_{B}=0.0008$, $t_{a} / \omega_{B}=10, t_{b} / \omega_{B}=1$. 
In Figure 6, we plot the dynamics of mean number of photons. Again, we see the mutual influence of $\mathrm{RO}$ and $\mathrm{BO}$. For dc field absence (Figure $6 \mathrm{~b}$ ), the collapse-revivals scenario corresponds the Jaynes-Cummings model for single atom [26]. It represents the quantum interference of the spectral components with different Rabi-frequencies $\Omega_{n}$ and different relative amplitudes [26]. For dc field appearance (Figure 6a), there is one more type of interference components with frequencies $m \omega_{B}(m$ is integer value). Among them, the term with $m=1$ is dominant, and its amplitude is comparable with RO components. As a result, the mean number of photons is modulated with Bloch frequency $\omega_{B}$. Therefore, the dc field via BO opens one more way of engineering of the quantum light states. This effect depends on the large number of physical factors, such as dc field value, energy and dipole moment of quantum transition for the atom, tunnel coupling, etc. Concluding, the results of numerical modeling agree with the simple analytical model, developed in Section 3. They are promising for different applications in quantum computing, quantum informatics, etc.
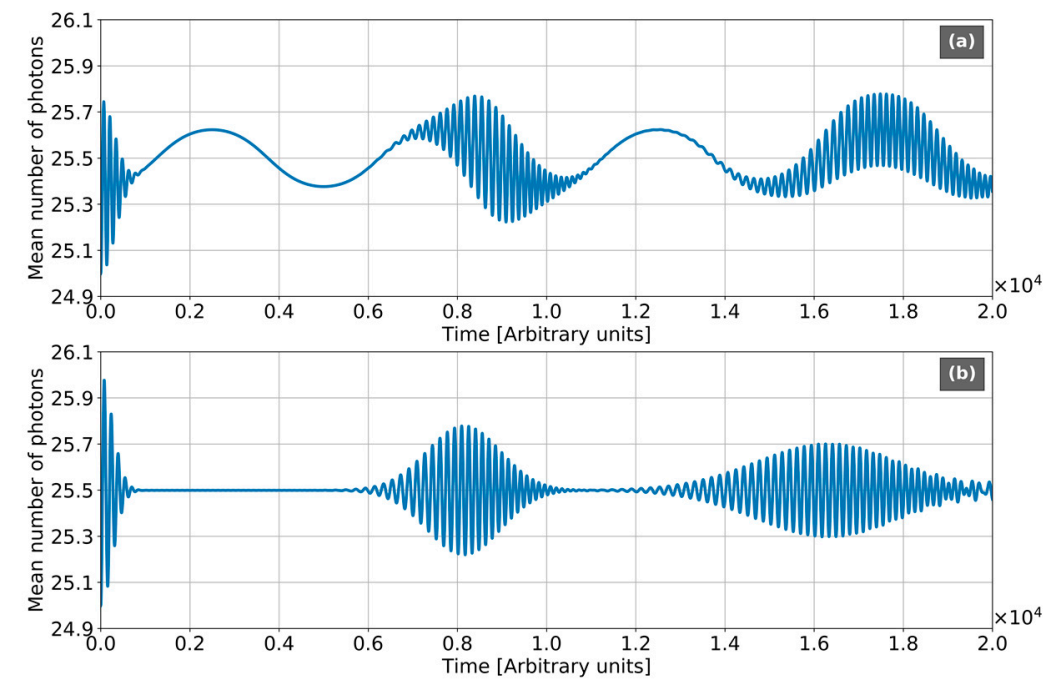

Figure 6. Mean number of photons for light in the coherent state with $\langle n\rangle=25$. All frequencies are normalized to the frequency of quantum transition $\omega_{0}, \Omega_{\langle n\rangle}=0.025$. Sampling frequency $=4$. (a) The chain with the number of atoms $N=128, \omega_{B}=0.0008$, electron initial Gaussian with $a_{0}=1, b_{0}=0, u_{a}=80, \sigma_{a}=10, k_{a}=0, t_{a} / \omega_{B}=10, t_{b} / \omega_{B}=1$. (b) Dressed single atom (Jaynes-Cummings model).

Figure 7 shows the quantum-statistical properties of light. The temporal behavior of the mean number of photons (Figure 7a) may be rather simply understood from the quasi-classical point of view developed in Section 3. The processes of photons emission and absorption correspond to the rapid jumps from value $\langle n\rangle \approx 0.1$ to $\langle n\rangle \approx 0.9$ (and vice versa). Comparing Figure 7a with Figure $4 \mathrm{c}$, one can see these jumps occurrence in a small vicinity of the stops and turns of the wavepacket. The neighboring stopping points are separated in time by half of a Bloch circle. Sufficiently far from a stopping point, the motion is characterized by rather small acceleration. It means the existence of rather weak fluctuations of photonic number, dictated by $\mathrm{RO}$ and taking place with Rabi-frequency $\Omega_{0}=2 g$. This scenario dramatically differs from RO in the single atom, where the acts of photons emission-absorption correspond the harmonic oscillations with Rabi-frequency.

Figure $7 \mathrm{~b}$ displays the evolution of photonic variance. As it was mentioned above, the electrons and photons in the initial state are uncorrelated. Thus, electron-photon entanglement is poor at the intervals of motion between the stopping points, whereby the variance oscillates only due to photon number fluctuations of rather small amplitude with vacuum Rabi-frequency. The processes of photons emission-absorption are accompanied the strong narrow peaks of variance. Thus, the vicinities of stopping points are characterized by the strong non-classicality of light and high degree of its 
entanglement with electronic sub-system. This scenario strongly differs from the case of the harmonic oscillations of variance with Rabi-frequency for the single atom [26]. The obtained results show that such manifestations of non-classicality of light, such as electron-photon entanglement and accumulated entropy become controllable by dc field via BO. As it is well known [26], the physical origin of vacuum $\mathrm{RO}$ is a manifestation of spontaneous emission of the single exited state in strong coupling regime. The obtained dynamics qualitatively support the conclusion of an early paper [68], that BO dramatically changes the physical picture of spontaneous emission in the atomic chains.

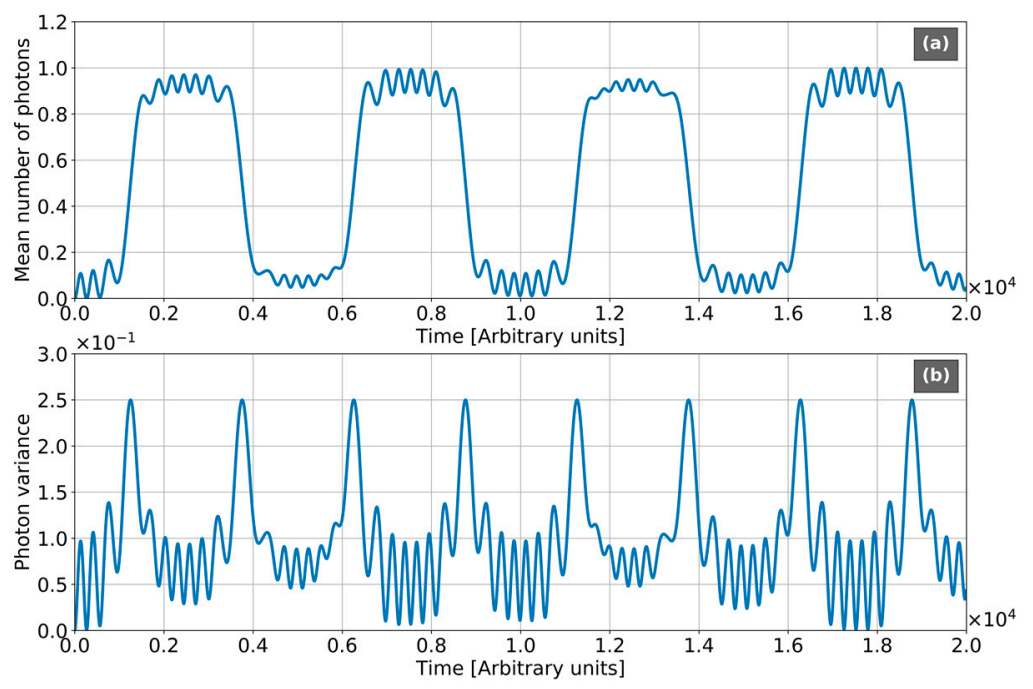

Figure 7. Quantum-statistical characteristics of light in the vacuum state. All frequencies are normalized to the frequency of quantum transition $\omega_{0}$, Sampling frequency $=4$, Rabi-frequency $\Omega_{0}=0.025$, the chain with the number of atoms $N=128, \omega_{B}=0.0008$, electron initial Gaussian with $a_{0}=1, b_{0}=0$, $u_{a}=80, \sigma_{a}=10, k_{a}=0, t_{a} / \omega_{B}=10, t_{b} / \omega_{B}=1$. (a) Mean number of photons. (b) Photonic variance.

To illustrate the mutual influence of high-frequency and low-frequency motions, we show the spatial-temporal evolution of inversion for initial condition (34) (Figure 8). One finds out of the phase oscillations of inversion with Bloch frequency. The two halves of every Bloch period correspond to the excited and ground state, respectively. Here, $\mathrm{BO}$ of inversion is modulated with RO. Such dynamics are similar to the shown for the vacuum initial state at Figure $4 \mathrm{c}$, however, the modulation depth strongly increases due to the influence of initial entanglement (the inversion evolves over the single Rabi-circle between zero and \pm 1 ). In Figure 9, we show the tunnel current for initial state (34), which as well as inversion exhibits the high level of $\mathrm{RO}$ modulation. Comparing it with Figure $5 \mathrm{c}$, one finds again the strong influence of initial electron-photon correlations.

Figure 10 displays the quantum-statistical properties of light. The evolution of mean number of photons (Figure 10a) may be considered as a periodic system of step-like beatings (dashed line), modulated with high-frequency oscillations (solid curve). The value $\langle n\rangle$ averaged with respect to the step-period is approximately equal to 0.5 , which is equal to the main photon number for both states $\left|\Psi_{n 1,2}\right\rangle$ given by Equations (20) and (21). The general physical interpretation of Figure 10a may be done from the quasi-classical point of view as an interference of this states (of course, we speak here about moving wavepackets, but not about perfect travelling waves). Again, the photon emission-absorption with Bloch-period takes place in the vicinity of stopping points. One can see, comparing Figure 10a with Figure 9, that the moments of shut-down are agreed with zeros of inversion. Translatory motion of the wavepacket is accompanied by RO between eigenstates, which correspond to high-frequency fluctuations with Rabi-frequency. In Figure 10b we plot the dynamics of photonic variance. In contrast with vacuum photonic state, the variance is maximal at the intervals of translatory motion and strongly decreases in the vicinities of stopping points. The reason for it is that translatory motion corresponds 
to the statement in one of the Eigenstates (20) and (21). Every one of these states is characterized by the maximal level of electron-photon entanglement. Photons emission-absorption means the mutual transformation of the States (20) and (21). It leads to creation the coherent superposition of these states for a short time with comparable probability amplitudes. It leads to the breaking of electron-photon correlations. Again, RO-fluctuations are imposed to this ideal picture similar to the case of the vacuum photonic state. The photonic variance in these oscillations reaches the value $\delta n=0.25$, which corresponds to the maximal one for two States (20) and (21).

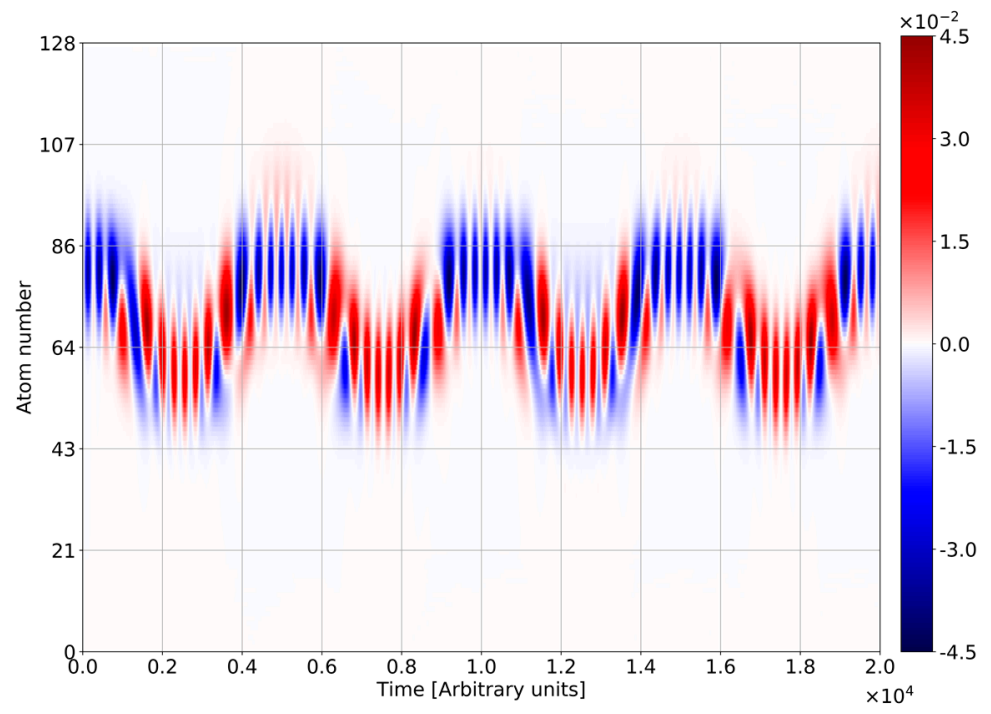

Figure 8. Space-time distribution of inversion density for the case of atom-light initial entanglement. All frequencies are normalized to the frequency of quantum transition $\omega_{0}$. Sampling frequency $=4$. The number of atoms $N=128$, Rabi-frequency $\Omega_{0}=0.025, \omega_{B}=0.0008$, electron initial Gaussian with $a_{0}=1, b_{0}=0, u_{a}=80, \sigma_{a}=10, k_{a}=0, t_{a} / \omega_{B}=10, t_{b} / \omega_{B}=1$.

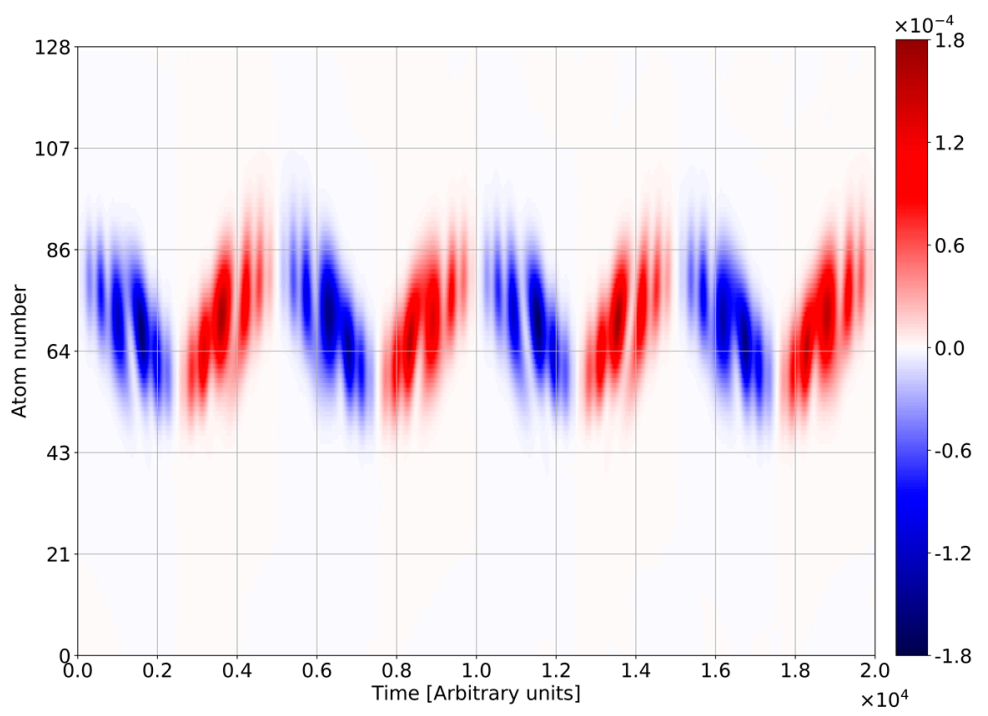

Figure 9. Space-time distribution of tunnel current density for the case of atom-light initial entanglement. All frequencies are normalized to the frequency of quantum transition $\omega_{0}$. Sampling frequency $=4$. The number of atoms $N=128$, Rabi-frequency $\Omega_{0}=0.025, \omega_{B}=0.0008$, electron initial Gaussian with $a_{0}=1, b_{0}=0, u_{a}=80, \sigma_{a}=10, k_{a}=0, t_{a} / \omega_{B}=10, t_{b} / \omega_{B}=1$. 

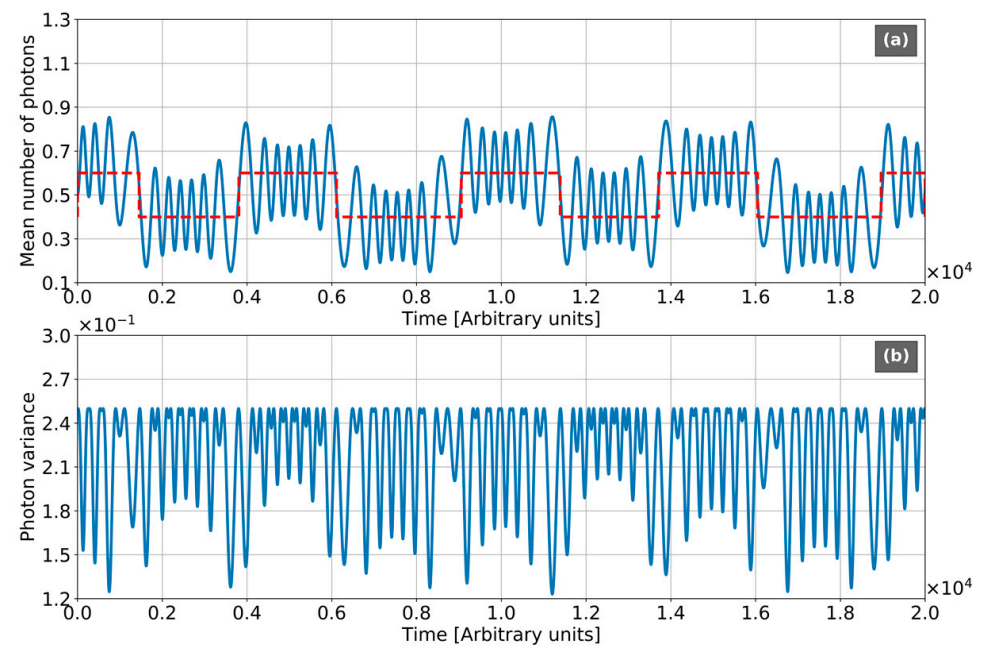

Figure 10. Quantum-statistical characteristics of light in the entangled initial state. Mean number of photons for the case of atom-light initial entanglement. All frequencies are normalized to the frequency of quantum transition $\omega_{0}$. Sampling Frequency $=4$. Rabi-frequency $\Omega_{0}=0.025$, the chain with number of atoms $N=128, \omega_{B}=0.0008$, electron initial Gaussian with $a_{0}=1, b_{0}=0, u_{a}=80, \sigma_{a}=10, k_{a}=0$, $t_{a} / \omega_{B}=10, t_{b} / \omega_{B}=1$. (a) Mean number of photons. (b) Photonic variance.

\section{Some Other Potential Experimental Implementations}

\subsection{Semiconductor $2 D$-Heterostructures}

The next promising candidate for the experimental implementation of the model considered above is the low-dimensional semiconducting heterostructure (see Figure 2) under femtosecond inter-sub-band excitation [50]. For example, the sample consisting of $51 \mathrm{GaAs}$ quantum wells separated by barriers was implemented in [50]. A coherent excitation of the sample was created by a femtosecond pulse with a center frequency resonant to the inter-sub-band transition. The resonant line at $30 \mathrm{THz}$ was homogeneously broadened with coherence time $320 \mathrm{Fs}$. The amplitude of incident field was varied inside of area $(5-50) \mathrm{kV} / \mathrm{cm}$. As a result, there were observed coherent sub-picosecond $\mathrm{RO}$ and manipulated in a wide range by varying the strength of the coherent driving field. The measurements [50] qualitatively agreed with the predictions of the simplest model based on Maxwell-Bloch equations for non-interactive two-level systems. Conventional simplifications, such as RWA and omitting of all types of damping have been used. The simulation of the pulse driving field was done by varying the Rabi frequency $\Omega_{R}=\Omega_{R}(t)$.

Here, we add the dc field for manipulation by atomic chain via BO. For the application of our model to the potential experiments with 2D-heterostructures, it is necessary to describe the driving quantum light as a short transient normally incident to the heterostructure. The driving process in this case is described by the instantaneous coupling coefficient $g$. The field quantization should be modified following Appendix $C$. The quantum properties of light in the wavepacket are dictated by the special pair of bosonic creation-annihilation operators, which allows us to rewrite the dynamic Equations (7) and (8) as:

$$
\begin{gathered}
i \hbar \frac{\partial a_{p, n}}{\partial t}=\left(\delta \varepsilon-\mathbf{E}_{d c} \cdot e \mathbf{R}_{p}\right) a_{p, n}+t_{a} a_{p+1, n}+t_{a}^{*} a_{p-1, n}-g(t) \sqrt{n+1} b_{p, n+1}, \\
i \hbar \frac{\partial b_{p, n+1}}{\partial t}=-\left(\delta \varepsilon+\mathbf{E}_{d c} \cdot e \mathbf{R}_{p}\right) b_{p, n+1}+t_{b} b_{p+1, n+1}+t_{b}^{*} b_{p-1, n+1}-g(t) \sqrt{n+1} a_{p, n},
\end{gathered}
$$

where $g(t)=\mathbf{d}_{a b} \cdot \mathbf{u}_{0}(t) / \hbar, \mathbf{u}_{0}(t)$ is a slow envelope of the driving pulse.

The driving with electromagnetic pulse leads to chain dynamics qualitatively different from the case of monochromatic light. Figures 11 and 12 depict the temporal-spatial evolution of inversion 
for different relations between barrier penetrations at $1 S$ and $2 S$ levels. Figure 11 shows the case of identical penetrations. One finds $\mathrm{BO}$ over the pulse duration. The oscillations are modulated with Rabi frequency and stop with the pulse disappearing at zero inversion. The behavior becomes quite different for the case of $2 S$ penetration strongly exceed $1 S$ one. (Figure 12). No oscillations exist before the pulse appearance $(t<0.9)$. The reason for this is a weak value of penetration at $1 S$ level. The switching of pulse creates both $\mathrm{RO}$ and $\mathrm{BO}$. The $\mathrm{RO}$ existence is limited by the pulse duration $(t<1.2)$, while BO continues even after the pulse switches off. It is a result of tunneling at the 2S-level on which the penetration is much higher. The charge existence at the 2S-level is a result of resonant pumping by the optical pulse. Thus, we can consider the resonant pumping as a tool of $\mathrm{BO}$ triggering and speak about photon-assisted BO.

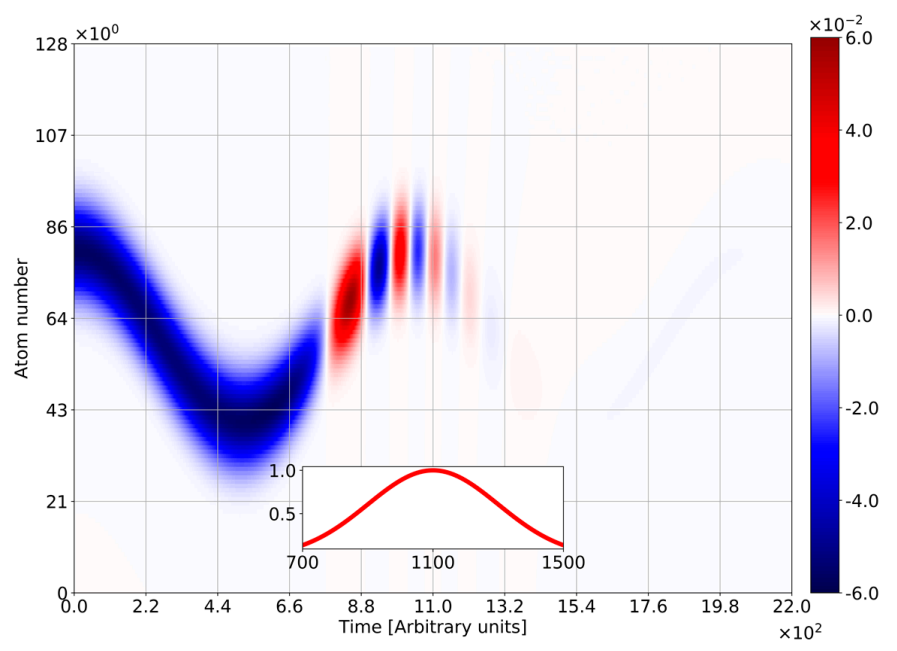

Figure 11. Space-time distribution of inversion density for pulse light in the coherent state with $\langle n\rangle=25$. All frequencies are normalized to the frequency of quantum transition $\omega_{0}$. Sampling frequency $=20$. The number of atoms $N=128$, Rabi-frequency $\Omega_{\langle n\rangle}=0.2$, electron initial Gaussian with $a_{0}=1, b_{0}=0, u_{a}=80, \sigma_{a}=10, k_{a}=0, t_{a} / \omega_{B}=t_{b} / \omega_{B}=10$. Insert: the envelope of the light pulse in the time scale matched with the main picture.

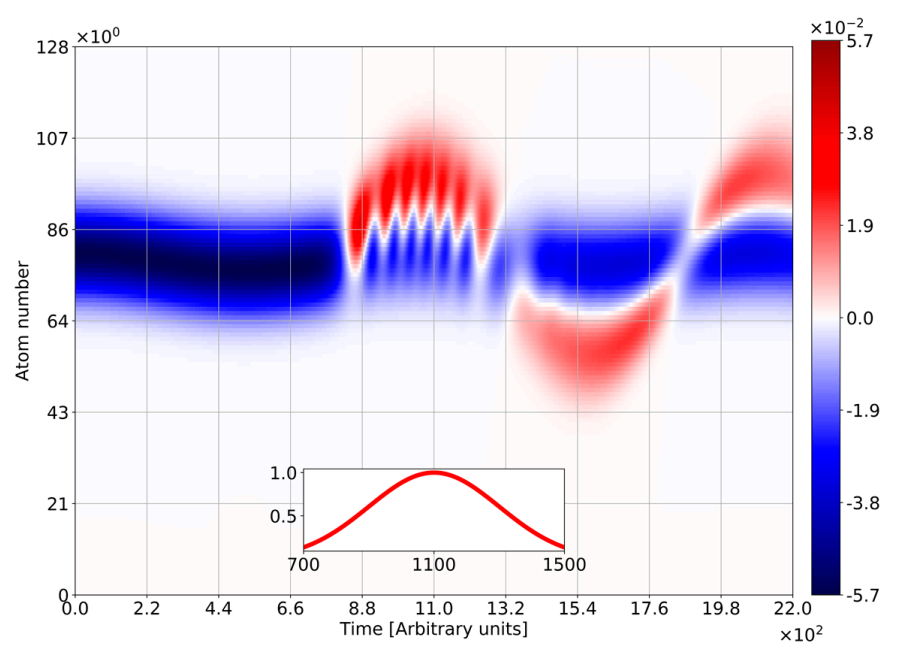

Figure 12. Space-time distribution of inversion density for pulse light in the coherent state with $\langle n\rangle=25$. All frequencies are normalized to the frequency of quantum transition $\omega_{0}$. The number of atoms $N=128$, Rabi-frequency $\Omega_{\langle n\rangle}=0.2$, electron initial Gaussian with $a_{0}=1, b_{0}=0, \omega_{B}=0.02$, $u_{a}=80, \sigma_{a}=10, k_{a}=0, t_{a} / \omega_{B}=10, t_{b} / \omega_{B}=1$. Insert: the envelope of the light pulse in the time scale matched with the main picture. 


\subsection{Josephson Junction}

The next promising candidate for experiment implementation is a single Josephson junction embedded in an inductive environment. As it was shown in [53-56], the voltage-biased Josephson junction exhibits the temporal dynamics equal to the motion of a fictitious charged particle with inertia provided by the inductance. This particle moves in a potential, which also has a periodic part and a linear tilt. On the other hand, a Josephson junction strongly coupled to the microwave resonator (Figure 3) in the resonant regime may be considered as an artificial two-level atom ("fluxonium" [54]). It is described by the Jaynes-Cummings model [54] and exhibits RO dynamics [54]. Here, we will focus on the case of Josephson junction simultaneously driven by dc voltage and quantum light in the regime of small detuning. We will show that the model investigated in this work is applicable to this type of physical system. Therefore, we will start our analysis by identifying the parameters of Josephson junction in terms of this model.

The temporal dynamics of entire circuit may be described using so-called quasi-charge representation $[53,55]$. Let us first consider the single junction, of which the standard form reads $\hat{H}_{0}=\left(\hat{Q}^{2} / 2 C_{J}\right)-E_{J} \cos \hat{\varphi}_{J}$, where $\hat{Q}=(2 e / i) \partial / \partial \hat{\varphi}_{J}, \hat{\varphi}_{J}$ are operators of junction charge and phase difference across the junction, respectively. It describes the particle of mass $C_{J}$ moving in the periodic potential [55] $\left(C_{J}\right.$ is a junction capacitance). The eigenfunctions of this Hamiltonian are Floquet-Bloch modes with condition of quasi-periodicity $\Psi_{\alpha, Q}(\varphi)=P_{\alpha, Q}(\varphi) e^{i \varphi Q / 2 e}$, where $P_{\alpha, Q}(\varphi)=P_{\alpha, Q}(\varphi+2 \pi)$ is periodic function, $\alpha$ is a number of the gap. These functions describe the motion of the quasiparticles, which is similar to the motion of electrons in crystal. The phase $\varphi$ and quasi-charge $Q$ play the role of the conjugated spatial coordinate and quasi-momentum for electrons in crystals, respectively. The energies of the two first modes $\varepsilon_{0,1}(Q)$, are separated by the gap, which in the regime of interest here $\left(E_{J}>>E_{C_{J}}, E_{C_{J}}=e^{2} / 2 C_{J}\right)$ is of the order of plasma frequency $\omega_{p}=\sqrt{8 E_{J} E_{C_{J}}} / \hbar$. Taking $E_{J} / h=30 \mathrm{GHz}$, and $E_{C_{J}} / h=3$, we have $\omega_{p}=150 \mathrm{GHz}$. Next, in the limit $E_{J}>>E_{C_{J}}$ the energy bands $\varepsilon_{0,1}(Q)$ are purely sinusoidal [53] with the bandwidths of the order of [55]

$$
\Delta_{0}=16 \sqrt{E_{C_{J}} E_{J} / \pi}\left(E_{J} / 2 E_{C_{J}}\right)^{1 / 4} e^{-\sqrt{8 E_{J} / E_{C_{J}}}}
$$

(the typical values are $\Delta_{0} \approx E_{C} / 2=1,5 \mathrm{GHz}$ ). The situation is the same in the case of atomic chain considered above (see Equation (13) in the case $g_{n}=0$ ). Thus, we are able to establish the correspondence $2 t_{a, b} \leftrightarrow \Delta_{0}$. $\hat{H}_{0}$ to

The additional series inductance and switching on the bias voltage transforms the Hamiltonian

$$
\hat{H}_{A}=\hat{Q}^{2} / 2 C_{J}+E_{L_{J}}\left(\phi_{b}-\hat{\varphi}_{J}\right)^{2} / 2+E_{J} \cos \left(\hat{\varphi}_{J}\right),
$$

where $E_{L_{J}}=(\hbar / 2 e)^{2} L_{2}^{-1}$ is the constant of inductance energy, $\phi_{b}$ is the total phase difference across the circuit, which is determined by the bias voltage $V_{b}$, such that $\dot{\phi}_{b}=2 e V_{b} / \hbar$ [53].

The case under consideration has a simple physical interpretation based on the charge-phase duality [53]. The voltage-biased junction with inductive environment and the current-biased junction shunted by a capacitor $C_{J}$ are dual to each other when exchanging the role of quasi-charge and phase. The dynamics of the circuit, shown in Figure 3, are equal to the intra-band motion of a fictitious charged particle in the tilted periodic potential. The tilt leads to $\mathrm{BO}$ of the particle in the quasi-charge space, which allows for the identification of the Bloch-frequency as dual plasma frequency $\omega_{B} \Leftrightarrow \omega_{c}=\pi\left(\Delta_{0} L_{2}\right)^{1 / 2} / e$.

The inductive strong coupling of a Josephson junction with a microwave resonator leads to its dressing with microwave photons. It corresponds to the periodic emission-absorption of photons and inter-band transitions by the fictitious charged particle (RO). Thus, the qualitative picture of junction behavior is similar to the BO of dressed electrons considered above: The periodic inter-band transitions ( $\mathrm{RO}$ of fluxonium) are accompanied by the charge oscillations through the bias-voltage 
driving. For formal identification of the coupling parameters of the system, "junction + microwave resonator", it is necessary to analyze in more detail the total Hamiltonian. It reads

$$
\hat{H}=\hat{H}_{A}+\hat{H}_{p h}+\hat{H}_{I, p h}+\hat{H}_{I, d c}
$$

where $\hat{H}_{A}$ is a Hamiltonian of the Josephson junction in an inductive environment, $\hat{H}_{p h}$ is Hamiltonian of microwave photons in the resonator, $\hat{H}_{I, p h}$ is a Hamiltonian of junction-cavity interaction, and $\hat{H}_{I, d c}$ is a support of biased voltage. For further analysis, it is convenient to use the secondary quantization technique. The Hamiltonian of photons reads

$$
\hat{H}_{p h}=\hat{\Phi}_{r}^{2} / 2 L_{r}+\hat{Q}_{r}^{2} / 2 C_{r}
$$

where $\hat{Q}_{r}=i \sqrt{\frac{\hbar}{2 Z_{r}}}\left(\hat{a}-\hat{a}^{+}\right), \hat{\Phi}_{r}=i \sqrt{\frac{\hbar Z_{r}}{2}}\left(\hat{a}+\hat{a}^{+}\right), \hat{a}, \hat{a}^{+}$are creation-annihilation operators for microwave photons, which satisfy the conventional bosonic commutative relations. By following the concept of quasi-charge-phase duality, the Fourier-Bloch states $\Psi_{\alpha, Q}(\varphi)$ exchanged by the Wannier functions (see Appendix A) and the Hamiltonian is written in the Wannier-basis. We denote the two first states with quasi-charge $Q$ as $\left|e_{Q}\right\rangle$ and $\left|g_{Q}\right\rangle$ and introduce the raising operators $\hat{\sigma}_{Q}=\left|e_{Q}\right\rangle\left\langle g_{Q}\right|$ and $\hat{\sigma}_{Q}^{+}=\left|g_{Q}\right\rangle\left\langle e_{Q}\right|$. The atomic Hamiltonian may be rewritten as $\hat{H}_{A}=\hbar \omega_{p} \sum_{Q} \hat{\sigma}_{Q z} / 2$, where $\hat{\sigma}_{Q z}$ is Pauli inversion matrix.

For junction-cavity interaction we have [54]

$$
\hat{H}_{I}=\frac{2 e}{\hbar} G \hat{\Phi}_{r} \phi_{e g} \sum_{Q}\left(\hat{\sigma}_{Q}^{+}+\hat{\sigma}_{Q}^{-}\right)
$$

where $G \approx(\hbar / 2 e)^{2} L_{1}\left(L_{2} L_{r}\right)^{-1}, \phi_{e g}=\left\langle g_{Q}\left|\hat{\sigma}_{Q}^{+}\right| e_{Q}\right\rangle$ is the matrix element of effective dipole moment. The Hamiltonian is written in rotating-wave approximation. It is equal to the Hamiltonian (3) if the coupling coefficient identified with the value

$$
g \Leftrightarrow \frac{\left|\phi_{e g}\right|^{2}}{\sqrt{2}} \sqrt{\frac{R_{0}}{2 \pi Z_{r}}} \frac{L_{1}}{L_{2}} \omega_{p},
$$

where $R_{0}$ is the quantum of resistance. The validity of rotating-wave approximation implies all relevant energies to be smaller than the plasmonic frequency of the Josephson junction and resonant frequency of resonator. In particular, we impose the characteristic energy, $E_{L_{J}}$, associated with the inductance $L_{2}$ to be smaller than $\hbar \omega_{p}$. It corresponds to the values $L_{2} \approx 200 \mathrm{nH}$, which are quite reachable [56]. Second, we need also $g<<\omega_{p}$. For standard value $Z_{r}=50 \mathrm{k} \Omega$ [56] we have $L_{1} / L_{2} \approx 10^{-3}$ for this condition been imposed. The coherence time and energy relaxation time both are of the order $\cong \mu \mathrm{s}[56]$. Thus, the number of $\mathrm{BO}$ flops is high enough for their experimental observation in the fluxonium atom in microwave frequency range. The real cases may be described by lossless model considered above.

\section{Conclusion and Outlook}

In this paper, we developed a model of the chain of two-level artificial atoms manipulated simultaneously by a dc field and single-mode quantum light in the strong coupling regime. Atom-light interactions were assumed to be resonant. The cases of monochromatic light and light pulse have been considered. Both, electronic and photonic characteristics of the oscillation process, have been studied, such as: (i) inversion density; (ii) tunneling current density; (iii) distribution of photon probabilities; (iv) mean number of photons; (v) photon number variance; and (vi) Neumann entropy of light. The different types of the initial states of the light have been considered: (i) coherent state; (ii) vacuum photonic state; and (iii) Fock-state entangled with the electron wave packet. The Gaussian wavepackets were chosen as initial states of atoms. 
The model is universal with respect to the physical origin of artificial atoms and frequency ranges of atom-light interaction. The model was adapted to the semiconductor 2D-heterostructures (THz frequencies), semiconductor quantum dots (optical range), and Josephson junctions (microwaves). The initial data for numerical simulations are taken from recently published experiments.

The dynamical equations have been studied both analytically and numerically. The idea for analytical simplification is based on the separate description of electrons dressing with photons and quasi-classical motion of their geometrical center driven by a dc field. The analytical solutions are in good qualitative agreement with numerical simulations. Our model is based on such conventional simplifications as neglect of damping and RWA. Their validity for considered systems was supported by numerical estimations taken from experimental data.

The following conclusions are emerged from our studies:

(1) The case of initial coherent state of the light exhibits the collapse-revival picture, which drifts over the chain (collapses and revivals placed in the different spatial areas). In contrast with Jaynes-Cummings model, the collapse-revival picture is modulated with Bloch frequency;

(2) In the case of initial vacuum state of light, the photon emission and absorption occurs with Bloch frequency, instead of Rabi-frequency in Jaynes-Cummings model. The photonic probabilities are mainly modulated with Bloch frequency, while the contribution of Rabi-components is rather slight. BO strongly squeezes the vacuum state of light entangled with electronic wavepackets;

(3) The electron-photon entanglement dramatically modifies the tunnel current behavior. It becomes modulated agreed with the collapse-revival picture for the case of coherent state of light, and periodically modulated by $\mathrm{RO}$ for the case of initial photonic vacuum;

To conclude, the main result of the paper is the novel effect of the influence of $\mathrm{BO}$ and quantum statistical properties of light on each other. It is counterintuitive because of the strongly different frequency ranges for such types of oscillations existence. The reason for it is an entanglement of electronic and photonic states in the system been considered.

The obtained results allow for the control of quantum-statistical properties of light via adiabatically turning the dc field. They are promising for applications in quantum optics, quantum informatics, and quantum computing. The mutual modulation of low-frequency $\mathrm{BO}$ and high-frequency inter-band transitions produces the new types of spectral lines in tunneling current and optical polarization. It opens the promising ways in spectroscopy of nanodevices in $\mathrm{THz}$ and optical frequency ranges. These problems may be considered as directions for future research activity.

Author Contributions: Developments of the physical models, derivation of the basis equations, interpretation of the physical results and righting the paper have been done by I.L. and G.S. jointly. The Numerical Python calculations and Matplotlib figures were produced by I.L.

Acknowledgments: G.S. acknowledges support from the project FP7-PEOPLE-2013-IRSES-612285 CANTOR. We gratefully acknowledge the stimulating discussions with Professor Dmitry Mogilevtsev.

Conflicts of Interest: The authors declare no conflicts of interest.

\section{Appendix A. Derivation of Hamiltonian in the Wannier-Fock Basis}

The Hamiltonian of conductive electron movement in the chain associated with the interatomic tunneling, which is described by the periodic potential $V(\mathbf{r})$, is

$$
\hat{H}_{0}=\frac{\hat{\mathbf{p}}^{2}}{2 m}+V(\mathbf{r})
$$

The eigenmodes of Hamiltonian (A1) are two Bloch modes correspondent to valence $(b)$ and conductive (a) zones and denoted as $\psi_{\alpha, h}(\mathbf{r})=|\alpha, h\rangle, \alpha=a, b, h$ being a scalar quasi-momentum 
directed along the chain. Because the electrons are strongly confined inside the atoms, we will use as a basis, Wannier functions $\phi_{\alpha, \mathbf{R}_{j}}(\mathbf{r})=\left|\alpha_{j}\right\rangle$ [69], defined as a linear combinations of Bloch modes

$$
\phi_{\alpha, \mathbf{R}_{j}}(\mathbf{r})=\frac{1}{\sqrt{N}} \sum_{h} e^{-i h\left(\mathbf{e} \cdot \mathbf{R}_{j}\right)} \psi_{\alpha, h}(\mathbf{r}) .
$$

Let us mention some properties of Wannier functions, which are important for our analysis. Wannier function $\phi_{\alpha, \mathbf{R}_{j}}(\mathbf{r})$ is strongly localized inside the $j$-th atom. Inversion of Equation (A2) gives the expression of Bloch states in the terms of Wannier functions:

$$
\psi_{\alpha, h}(\mathbf{r})=\frac{1}{\sqrt{N}} \sum_{\mathbf{R}_{j}} e^{i h\left(\mathbf{e} \cdot \mathbf{R}_{j}\right)} \phi_{\alpha, \mathbf{R}_{j}}\left(\mathbf{r}-\mathbf{R}_{j}\right)
$$

Wannier functions satisfy the orthogonality relation $\left\langle\alpha_{j} \mid \alpha_{l}\right\rangle=\delta_{\alpha \alpha^{\prime}} \delta_{j l}$.

Projecting $\hat{H}_{0}$ on the Bloch modes and using (A3), we obtain the dispersion law for free-tunneling electron in the next form:

$$
\varepsilon_{\alpha}(h)=\left\langle\alpha, h\left|\hat{H}_{0}\right| \alpha, h\right\rangle=\frac{1}{N} \sum_{j, l} e^{-i h \mathbf{e} \cdot\left(\mathbf{R}_{j}-\mathbf{R}_{l}\right)}\left\langle\alpha_{j}\left|\hat{H}_{0}\right| \alpha_{l}\right\rangle .
$$

Denoting

$$
\left\langle\alpha_{j}\left|\hat{H}_{0}\right| \alpha_{l}\right\rangle=\left\{\begin{array}{cc}
\varepsilon_{\alpha}(0), & j=l \\
t_{\alpha}\left(\mathbf{R}_{j}-\mathbf{R}_{l}\right), & j \neq l
\end{array},\right.
$$

we can rewrite (A4) in the following form:

$$
\varepsilon_{\alpha}(h)=\varepsilon_{\alpha}(0)+\frac{1}{N} \sum_{\substack{j, l \\ j \neq l}} e^{-i h \mathbf{e} \cdot\left(\mathbf{R}_{j}-\mathbf{R}_{l}\right)} t_{\alpha}\left(\mathbf{R}_{j}-\mathbf{R}_{l}\right) .
$$

The Hamiltonian (A1) may be presented with the Wannier basis in the form of a block diagonal matrix

$$
\hat{H}_{0}=\left(\begin{array}{cc}
\hat{H}_{0 a} & 0 \\
0 & \hat{H}_{0 b}
\end{array}\right)
$$

with diagonal-on elements

$$
\hat{H}_{0 \alpha}=\varepsilon_{\alpha}(0) \sum_{j}\left|\alpha_{j}\right\rangle\left\langle\alpha_{j}\left|+\sum_{\substack{j, l \\ j \neq l}} t_{\alpha}\left(\mathbf{R}_{j}-\mathbf{R}_{l}\right)\right| \alpha_{l}\right\rangle\left\langle\alpha_{j}\right|,
$$

where $\alpha=a, b$. The tunneling coupling exist only between the neighboring atoms (tight-binding approximation). Thus, we omit for brevity the spatial arguments in tunneling matrix elements: $t_{a, b}\left(\mathbf{R}_{j}-\mathbf{R}_{j+1}\right)=t_{a, b}, t_{a, b}\left(\mathbf{R}_{j}-\mathbf{R}_{j-1}\right)=t_{a, b}^{*}$. The Hamiltonian (A7) subject to (A8) reads

$$
\begin{aligned}
\hat{H}_{0}= & \varepsilon_{b}(0) \sum_{j}\left|b_{j}\right\rangle\left\langle b_{j}\left|+\varepsilon_{a}(0) \sum_{j}\right| a_{j}\right\rangle\left\langle a_{j}\right|+ \\
& \sum_{j}\left(t_{b}\left|b_{j}\right\rangle\left\langle b_{j+1}\left|+t_{b}^{*}\right| b_{j}\right\rangle\left\langle b_{j-1}\right|\right)+ \\
& \sum_{j}\left(t_{a}\left|a_{j}\right\rangle\left\langle a_{j+1}\left|+t_{a}^{*}\right| a_{j}\right\rangle\left\langle a_{j-1}\right|\right)
\end{aligned}
$$

Hamiltonian (A9) in the secondary quantization form is equivalent to the sum of intra-level term and tunneling Hamiltonian. 
The total Hamiltonian may be presented as $\hat{H}=\hat{H}_{0}+\hat{H}_{p h}+\hat{H}_{I, p h}+\hat{H}_{I, d c}$, where the second component $\hat{H}_{p h}=\hbar \omega \hat{a}^{+} \hat{a}$ corresponds to the free single-mode photons motion, and $\hat{a}, \hat{a}^{+}$are creation and annihilation operators of photons. We describe the atom-photon interaction in the dipole approximation by the third term $\hat{H}_{I, p h}=-e \hat{\mathbf{E}}(\mathbf{r}, t) \cdot \hat{\mathbf{r}}[26]$, where

$$
\hat{\mathbf{E}}=\mathbf{e} \sqrt{\frac{\hbar \omega}{2 \varepsilon_{0} V}}\left(\hat{a}+\hat{a}^{+}\right)
$$

is the single-mode quantum light operator of electric field, $V$ is the normalizing volume, and $\mathbf{e}$ is the unit polarization vector.

The quantum state of light will be described in terms of photons number states (Fock-states) $|m\rangle$. The basis states should be used in our analysis correspond to the independent photons number states and Wannier electrons $\left|\alpha_{j}, m\right\rangle$ (Wannier-Fock basis). Let us proceed to the calculation of matrix elements

$$
H_{I, p h}^{m n, l j, \alpha \alpha^{\prime}}=\left\langle m, \alpha_{l}\left|\hat{H}_{I, p h}\right| \alpha^{\prime}{ }_{j}, n\right\rangle=-e \sqrt{\frac{\hbar \omega}{2 \varepsilon_{0} V}}\left\langle\alpha_{l}\left|\mathbf{e} \cdot \hat{\mathbf{r}}_{j}\right| \alpha_{j}^{\prime}\right\rangle\left\langle m\left|\left(\hat{a}+\hat{a}^{+}\right)\right| n\right\rangle .
$$

Let us shift the origin for a given $j$ to the center of the $j$-th atom via the relation $\hat{\mathbf{r}}_{j}=\mathbf{R}_{j} \hat{I}+\hat{\mathbf{r}}^{\prime}$. Using the Wannier functions orthogonality for different electron bands, we have $\left\langle\alpha_{j}\left|\hat{\mathbf{r}}_{j}\right| \alpha^{\prime}{ }_{j}\right\rangle=\mathbf{R}_{j} \delta_{\alpha \alpha^{\prime}}+$ $\left\langle\alpha_{j}\left|\hat{\mathbf{r}}^{\prime}\right| \alpha^{\prime}{ }_{j}\right\rangle$. Together with orthogonality of the Fock states it makes (A11)

$$
H_{I, p h}^{m n, j l, \alpha \alpha^{\prime}}=-e \sqrt{\frac{\hbar \omega}{2 \varepsilon_{0} V}}\left(\sqrt{n} \delta_{m, n-1}+\sqrt{n+1} \delta_{m, n+1}\right) \begin{cases}{\left[\left(\mathbf{e} \cdot \mathbf{R}_{j}\right) \delta_{\alpha \alpha^{\prime}}+\left(\mathbf{e} \cdot \mathbf{d}_{\alpha \alpha^{\prime}}\right)\right],} & j=1 \\ \delta_{\alpha \alpha^{\prime}} \widetilde{c}_{j, \alpha \prime} & l=j+1 \\ 0, & \text { others }\end{cases}
$$

Here $\mathbf{d}_{\alpha \alpha^{\prime}}=\left\langle\alpha_{j}\left|\mathbf{r}_{j}\right| \alpha^{\prime}{ }_{j}\right\rangle$ is the matrix of the dipole moments, $\widetilde{d}_{j, \alpha}=\left\langle\alpha_{j}\left|\left(\mathbf{e} \cdot \mathbf{r}_{j}\right)\right| \alpha_{j+1}\right\rangle$. The second term at the top line characterizes quantum transitions in the separate atom. For real atoms (excluding hydrogen) only off-diagonal elements, $\mathbf{d}_{\alpha \alpha^{\prime}}$ are non-zero due to the inversion symmetry [70]. For some types of artificial atoms (such as quantum dots) the inversion symmetry is not obligatory. In this case on-diagonal elements of dipole matrix may be non-zero [37], which corresponds to the special type of intra-level motion [37]. The middle line in (A12) corresponds to the interaction of neighboring atoms stimulated by photons (photon-assistant tunneling over the same level).

The last term $\hat{H}_{I, d c}=-e \mathbf{E}_{d c}(\mathbf{r}, t) \cdot \hat{\mathbf{r}}$ describes the dipole interaction of the chain with the dc field. Its matrix elements are

$$
H_{I, d c}^{m n, l j, \alpha \alpha^{\prime}}=\left\langle m, \alpha_{l}\left|\hat{H}_{I, d c}\right| \alpha^{\prime}{ }_{j}, n\right\rangle=-e \delta_{m n} \mathbf{E}_{d c} \cdot\left\langle\alpha_{l}|\hat{\mathbf{r}}| \alpha^{\prime}{ }_{j}\right\rangle
$$

Using the orthogonality of Wannier states, we obtain

$$
H_{I, d c}^{m n, j l, \alpha \alpha^{\prime}}=-e \delta_{m n} \mathbf{E}_{d c} \begin{cases}\mathbf{R}_{j} \delta_{\alpha \alpha^{\prime}}+\mathbf{d}_{\alpha \alpha^{\prime}}, & j=l \\ \delta_{\alpha \alpha^{\prime}} \widetilde{d}_{j, \alpha} \mathbf{e}, & l=j+1 \\ 0, & \text { others }\end{cases}
$$

The first term in the top line corresponds to BO, the second one describes the Stark-effect [70]. 


\section{Appendix B. Derivation of Formula for Tunneling Current}

For calculating the tunneling current we introduce the operator of the particle number in the $j$-th atom $\hat{N}_{j}=\left|a_{j}\right\rangle\left\langle a_{j}|+| b_{j}\right\rangle\left\langle b_{j}\right|$ and formulate the equation of continuity

$$
\hat{J}_{j}-\hat{J}_{j-1}=-e \frac{d \hat{N}_{j}}{d t}
$$

where $\hat{J}_{j}$ is the current density operator in the $j$-th atom and the left-hand part in (A15) is a discrete analog of divergence in 1D-case. These operators act only to the electronic states; therefore all relations of this Appendix are given in terms of Wannier states. Using Heisenberg equation for the operator $\hat{N}_{j}$, we rewrite (A15) in the form

$$
\hat{J}_{j}-\hat{J}_{j-1}=-i \frac{e}{\hbar}\left[\hat{H}, \hat{N}_{j}\right] .
$$

The tunneling currents over excited and background energy levels are independent, thus $\hat{J}_{\text {Tunneling, } j}=\hat{J}_{\text {Tunneling, } j}^{(a)}+\hat{J}_{\text {Tunneling, }, j}^{(b)}$ Using (A16) we obtain

$$
\hat{J}_{\text {Tunneling, } j}^{(a, b)}-\hat{J}_{\text {Tunneling, } j-1}^{(a, b)}=-i \frac{e}{\hbar}\left[\hat{H}_{\text {Tunneling, }}^{(a, b)} \hat{N}_{j}\right],
$$

where

$$
\hat{H}_{\text {Tunneling }}^{(a)}=\sum_{j}\left(t_{a}\left|a_{j}\right\rangle\left\langle a_{j+1}\left|+t_{a}^{*}\right| a_{j}\right\rangle\left\langle a_{j-1}\right|\right)
$$

is a component of the Hamiltonian (2), chargeable for the tunneling at the excited level (the similar equation may be written for the Hamiltonian $\hat{H}_{\text {Tunneling }}^{(b)}$ ). Using (A18), we calculate the commutator in the right-hand part of (A17) and obtain

$$
\hat{J}_{\text {Tunneling, } j}^{(a)}-\hat{J}_{\text {Tunneling, } j-1}^{(a)}=- \text { iet }_{a}\left(\left|a_{j-1}\right\rangle+\left|a_{j+1}\right\rangle\right)\left\langle a_{j}\right|+\text { H.c. }
$$

It corresponds to the operator of the tunneling current at the excited level

$$
\hat{J}_{\text {Tunneling, } j}^{(a)}=- \text { iet }_{a}\left|a_{j}\right\rangle\left\langle a_{j+1}\right|+\text { H.c. }
$$

The observable value of the tunneling current is

$$
J_{\text {Tunneling, }, j}^{(a)}(t)=\left\langle\hat{J}_{\text {Tunneling, }, j}^{(a)}\right\rangle=-i e t_{a} a_{j}^{*}(t) a_{j-1}(t)+\text { c.c. }
$$

Using the approximation $a_{j-1}(t)-a_{j}(t) \approx\left(a_{j+1}(t)-a_{j-1}(t)\right) / 2$, and adding the similar support of the ground level, we obtain Relation (10). This relation corresponds to the well-known definition of the probability flow $\mathbf{j}=i \hbar\left(\Psi \operatorname{grad} \Psi^{*}-\right.$ c.c. $) / 2 m$ in $3 \mathrm{D}$ continuous case [70].

\section{Appendix C. Quantization of Electromagnetic Fields in Wavepackets}

For the secondary quantization in the wavepacket we use the method proposed by Keller [71]. First, we consider the linearly polarized electric field to have the spatial-temporal dependence for a 1D cavity resonator [26]. We assume the cavity formed by two parallel perfectly conductive planes distanced on the length $L$. The positive-frequency part of electric field operator is given [26]

$$
\hat{\mathbf{E}}^{(+)}(x, t)=\mathbf{e} \sum_{v} \sqrt{\frac{\hbar \omega_{v}}{2 \varepsilon_{0} V}} \hat{a}_{v} e^{i\left(k_{v} x-\omega_{v} t\right)},
$$

where $V$ is normalization volume, $k_{v}=v \pi / L, v$ is an integer value, $\omega_{v}=k_{v} c$. 
Next, following [71], we introduce the special complete system of basis classical wavepackets $\mathbf{u}_{\alpha}(x, t)$, every one of which is given by the superposition of cavity modes

$$
\mathbf{u}_{\alpha}(x, t)=\mathbf{e} \sum_{\beta} \sqrt{\frac{\hbar \omega_{\beta}}{2 \varepsilon_{0} V}} B_{\alpha \beta} e^{i\left(k_{\beta} x-\omega_{\beta} t\right)},
$$

where $B_{\alpha \beta}$ are elements of unit matrix with conventional unitary condition $\sum_{\gamma} B_{\gamma \alpha} B_{\gamma \beta}^{*}=\delta_{\alpha \beta}, \delta_{\alpha \beta}$ is the Kronecker delta. Equation (A22) using (A23) may be rewritten as

$$
\begin{gathered}
\hat{\mathbf{E}}^{(+)}(x, t)=\mathbf{e} \sum_{v} \sum_{v^{\prime}} \delta_{v v^{\prime}} \sqrt{\frac{\hbar \omega_{v}}{2 \varepsilon_{0} V}} \hat{a}_{v^{\prime}} e^{i\left(k_{v} x-\omega_{v} t\right)}= \\
\mathbf{e} \sum_{v} \sum_{v^{\prime}} \sum_{\gamma} B_{\gamma v^{\prime}} B_{\gamma v}^{*} \sqrt{\frac{\hbar \omega_{v}}{2 \varepsilon_{0} V}} \hat{a}_{v^{\prime}} e^{i\left(k_{v} x-\omega_{v} t\right)}= \\
\sum_{v} \sum_{\gamma} B_{\gamma v^{\prime}} \hat{a}_{v^{\prime}} \mathbf{u}_{\gamma}(x, t)
\end{gathered}
$$

Using (A24) and unitary condition, we obtain

$$
\hat{\mathbf{E}}^{(+)}(x, t)=\sum_{\gamma} \hat{c}_{\gamma} \mathbf{u}_{\gamma}(x, t)
$$

where $\hat{c}_{\gamma}=\sum_{v} B_{\gamma v} \hat{a}_{v}$ is a new set of creation-annihilation operators, satisfying the Bose commutative relations $\left[\hat{c}_{\alpha}, \hat{c}_{\beta}^{+}\right]=\delta_{\alpha \beta}$. The basis wavepackets are non-orthogonal:

$$
\int_{V} \mathbf{u}_{p}(x, t) \cdot \mathbf{u}_{q}^{*}(x, t) d V=\sum_{\alpha} \frac{\hbar \omega_{\alpha}}{2 \varepsilon_{0}} B_{p \alpha} B_{q \alpha}^{*} .
$$

The field Hamiltonian is

$$
\begin{gathered}
\hat{H}_{p h}=\varepsilon_{0} \int_{V}\left\{\hat{\mathbf{E}}^{(+)}(x, t) \cdot \hat{\mathbf{E}}^{(-)}(x, t)+\hat{\mathbf{E}}^{(-)}(x, t) \cdot \hat{\mathbf{E}}^{(+)}(x, t)\right\} d V= \\
\varepsilon_{0} \sum_{p} \sum_{q}\left(\hat{c}_{p} \hat{c}_{q}^{+}+\hat{c}_{q}^{+} \hat{c}_{p}\right) \int_{V} \mathbf{u}_{p}(x, t) \cdot \mathbf{u}_{q}^{*}(x, t) d V= \\
\sum_{\alpha} \frac{\hbar \omega_{\alpha}}{2} \sum_{p} \sum_{q} B_{p \alpha} B_{q \alpha}^{*}\left(\hat{c}_{p} \hat{c}_{q}^{+}+\hat{c}_{q}^{+} \hat{c}_{p}\right)
\end{gathered}
$$

Due to the property (A26), the energies of different modes are mixed via their mutual interference.

For applying the single mode approximation, some additional simplifications should be done. Let us assume that we consider the non-monochromatic field with the narrow frequency spectrum, which were localized in the vicinity of central frequency $\omega_{\alpha} \approx \widetilde{\omega}$. In this case the different modes $\mathbf{u}_{\alpha}(x, t)$ become approximately orthogonal and their interference disappears. The model of the wavepacket in the first order of dispersion theory gives

$$
\mathbf{u}_{p}(x, t)=\mathbf{u}(x, t) \approx \mathbf{u}_{0}\left(t-\frac{x}{v_{g r}}\right) e^{i(k(\widetilde{\omega}) x-\widetilde{\omega} t)},
$$

where $\mathbf{u}_{0}(t)$ is a slow envelope, the exponential factor gives the high-frequency filling, and $v_{g r}$ is a group velocity. The approximate field Hamiltonian and electric field operator read

$$
\begin{gathered}
\hat{H}_{p h} \approx \hbar \widetilde{\omega}\left(\hat{c}^{+} \hat{c}+\frac{1}{2}\right) ; \\
\hat{\mathbf{E}}(x, t) \approx \mathbf{u}_{0}\left(t-\frac{x}{v_{g r}}\right) \hat{c} e^{i(k(\widetilde{\omega}) x-\widetilde{\omega} t)}+\text { H.c. }
\end{gathered}
$$


respectively, where $\hat{c}^{+}, \hat{c}$ are annihilation-creation operators for photon in the wavepacket. The coupling factor $g=\mathbf{d}_{a b} \cdot \mathbf{u}_{0}(t) / \hbar$ becomes time-dependent, which allows us to consider the case of driving light in the form of the rather long laser pulse (assumed that the pulse duration strongly exceeds the period of high-frequency filling, which guarantees the RWA validity in our model).

\section{References}

1. Bloch, F. UË ber die Quantenmechanik der Elektronen in Kristallgittern. Z. Phys. 1929, 52, 555-600. [CrossRef]

2. Zener, C. A theory of electrical breakdown of solid dielectrics. Proc. R. Soc. Lond. 1934, 145, 523-529. [CrossRef]

3. Wannier, G.H. Wave functions and effective Hamiltonian for Bloch electrons in an electric field. Phys. Rev. 1960, 117, 432. [CrossRef]

4. Wannier, G.H. Stark ladder in solids? A reply. Phys. Rev. 1969, 181, 1364. [CrossRef]

5. Kuehn, W.; Gaal, P.; Reimann, K.; Woerner, M.; Elsaesser, T.; Hey, R. Coherent Ballistic Motion of Electrons in a Periodic Potential. Phys. Rev. Lett. 2010, 104, 146602. [CrossRef] [PubMed]

6. Waschke, C.; Roskos, H.; Schwedler, R.; Leo, K.; Kurz, H.; Köhler, K. Coherent submillimeter-wave emission from Bloch oscillations in a semiconductor superlattice. Phys. Rev. Lett. 1993, 70, 3319. [CrossRef] [PubMed]

7. Preiss, P.M.; Ma, R.; Tai, M.E.; Lukin, A.; Rispoli, M.; Zupanic, P.; Lahini, Y.; Islam, R.; Greiner, M. Strongly correlated quantum walks in optical lattices. Science 2015, 347, 1229-1233. [CrossRef] [PubMed]

8. Gluck, M.; Kolovsky, A.R.; Korsch, H.J. Wannier-Stark resonances in optical and semiconductor superlattices. Phys. Rep. 2002, 366, 103-182. [CrossRef]

9. Ben Dahan, M.; Peik, E.; Reichel, J.; Castin, Y.; Salomon, C. Bloch Oscillations of Atoms in an Optical Potential. Phys. Rev. Lett. 1996, 76, 4508. [CrossRef] [PubMed]

10. Madison, K.W.; Fischer, M.C.; Diener, R.B.; Niu, Q.; Raizen, M.G. Dynamical Bloch Band Suppression in an Optical Lattice. Phys. Rev. Lett. 1998, 81, 5093. [CrossRef]

11. Morsch, O.; Muller, J.H.; Cristiani, M.; Ciampini, D.; Arimondo, E. Bloch Oscillations and Mean-Field Effects of Bose-Einstein Condensates in 1D Optical Lattices. Phys. Rev. Lett. 2011, 87, 140402. [CrossRef] [PubMed]

12. Bongs, K.; Sengstock, K. Physics with Coherent Matter Waves. Rep. Prog. Phys. 2004, 67, 907. [CrossRef]

13. Ferrari, G.; Poli, N.; Sorrentino, F.; Tino, G.M. Long-Lived Bloch Oscillations with Bosonic Sr Atoms and Application to Gravity Measurement at the Micrometer Scale. Phys. Rev. Lett. 2006, 97, 060402. [CrossRef] [PubMed]

14. Battesti, R.; Cladé, P.; Guellati-Khélifa, S.; Schwob, C.; Grémaud, B.; Nez, F.; Julien, L.; Biraben, F. Bloch Oscillations of Ultracold Atoms: A Tool for a Metrological Determination of $\mathrm{h} / \mathrm{m}_{\mathrm{Rb}}$. Phys. Rev. Lett. 2004, 92, 253001. [CrossRef] [PubMed]

15. Morandotti, R.; Peschel, U.; Aitchison, J.S.; Eisenberg, H.S.; Silberberg, Y. Experimental Observation of Linear and Nonlinear Optical Bloch Oscillations. Phys. Rev. Lett. 1999, 83, 4576. [CrossRef]

16. Pertsch, T.; Dannberg, P.; Elflein, W.; Bräuer, A.; Lederer, F. Optical Bloch Oscillations in Temperature Tuned Waveguide Arrays. Phys. Rev. Lett. 1999, 83, 4752. [CrossRef]

17. Peschel, U.; Pertsch, T.; Lederer, F. Optical Bloch oscillations in waveguide arrays. Opt. Lett. 1998, 23, 1701-1703. [CrossRef] [PubMed]

18. Zheng, M.J.; Xiao, J.J.; Yu, K.W. Controllable optical Bloch oscillation in planar graded optical waveguide arrays. Phys. Rev. A 2010, 81, 033829. [CrossRef]

19. Bromberg, Y.; Lahini, Y.; Silberberg, Y. Bloch Oscillations of Path-Entangle Photons. Phys. Rev. Lett. 2010, 105, 263604. [CrossRef] [PubMed]

20. Afek, I.; Natan, A.; Ambar, O.; Silberberg, Y. Quantum state measurements using multipixel photon detectors. Phys. Rev. A 2009, 79, 043830. [CrossRef]

21. Afek, I.; Ambar, O.; Silberberg, Y. High-NOON States by Mixing Quantum and Classical Light. Science 2010, 328, 879-881. [CrossRef] [PubMed]

22. Sanchis-Alepuz, H.; Kosevich, Y.A.; Sanchez-Dehesa, J. Acoustic Analogue of Electronic Bloch Oscillations and Resonant Zener Tunneling in Ultrasonic Superlattices. Phys. Rev. Lett. 2007, 98, 134301. [CrossRef] [PubMed]

23. Anderson, B.P.; Kasevich, M.A. Macroscopic quantum interference from atomic tunnel arrays. Science 1998, 282, 1686-1689. [CrossRef] [PubMed] 
24. Kudo, K.; Monteiro, T.S. Theoretical analysis of super-Bloch oscillations. Phys. Rev. A 2011, 83, 053627. [CrossRef]

25. Hartmann, T.; Keck, F.; Korsch, H.J.; Mossmann, S. Dynamics of Bloch oscillations. New J. Phys. $2004,6,2$. [CrossRef]

26. Scully, M.O.; Zubairy, M.S. Quantum Optics; Cambridge University Press: Cambridge, UK, 2001.

27. Cohen-Tannoudji, C.; Dupont-Roc, J.; Grynberg, G. Atom-Photon Interactions: Basis Properties and Applications; Wiley: Chichester, UK, 1998.

28. Rabi, I.I. Space Quantization in a Gyrating Magnetic Field. Phys. Rev. 1937, 51, 652. [CrossRef]

29. Hocker, G.B.; Tang, C.L. Observation of the Optical Transient Nutation Effect. Phys. Rev. Lett. 1968, $21,591$. [CrossRef]

30. Johnson, T.A.; Urban, E.; Henage, T.; Isenhower, L.; Yavuz, D.D.; Walker, T.G.; Saffman, M. Rabi flopping between ground and Rydberg states with dipole-dipole atomic interactions. Phys. Rev. Lett. 2008, 100, 113003. [CrossRef] [PubMed]

31. Kamada, H.; Gotoh, H.; Temmyo, J.; Takagahara, T.; Ando, H. Exciton Rabi Oscillation in a Single Quantum Dot. Phys. Rev. Lett. 2001, 87, 246401. [CrossRef] [PubMed]

32. Blais, A.; Huang, R.-S.; Wallraff, A.; Girvin, S.M.; Schoelkopf, R.J. Cavity quantum electrodynamics for superconducting electrical circuits: An architecture for quantum computation. Phys. Rev. A 2004, 69, 062320. [CrossRef]

33. Gambetta, J.; Blais, A.; Schuster, D.I.; Wallraff, A.; Frunzio, L.; Majer, J.; Devoret, M.H.; Girvin, S.M.; Schoelkopf, R.J. Qubit-photon interactions in a cavity: Measurement-induced dephasing and number splitting. Phys. Rev. A 2006, 74, 042318. [CrossRef]

34. Blais, A.; Gambetta, J.; Wallraff, A.; Schuster, D.I.; Girvin, S.M.; Devoret, M.H.; Schoelkopf, R.J. Quantum-information processing with circuit quantum electrodynamics. Phys. Rev. A 2007, 75, 032329. [CrossRef]

35. Burkard, G.; Imamoglu, A. Ultra-long-distance interaction between spin qubits. Phys. Rev. B 2006, 74, 041307. [CrossRef]

36. Barrett, S.D.; Milburn, G.J. Measuring the decoherence rate in a semiconductor charge qubit. Phys. Rev. $B$ 2003, 68, 155307. [CrossRef]

37. Kibis, O.V.; Slepyan, G.Y.; Maksimenko, S.A.; Hoffmann, A. Matter Coupling to Strong Electromagnetic Fields in Two-Level Quantum Systems with Broken Inversion Symmetry. Phys. Rev. Lett. 2009, 102, 023601. [CrossRef] [PubMed]

38. Slepyan, G.Ya.; Yerchak, Y.D.; Maksimenko, S.A.; Hoffmann, A. Wave propagation of Rabi oscillations in one-dimensional quantum dot chain. Phys. Lett. A 2009, 373, 1374-1378. [CrossRef]

39. Slepyan, G.Ya.; Yerchak, Y.D.; Maksimenko, S.A.; Hoffmann, A.; Bass, F.G. Mixed states in Rabi waves and quantum nanoantennas. Phys. Rev. B 2012, 85, 245134. [CrossRef]

40. Yerchak, Y.; Slepyan, G.Y.; Maksimenko, S.A.; Hoffmann, A.; Bass, F. Array of tunneling-coupled quantum dots as a terahertz range quantum nanoantenna. J. Nanophotonics 2013, 7, 073085. [CrossRef]

41. Slepyan, G.Ya.; Yerchak, Y.D.; Hoffmann, A.; Bass, F.G. Strong electron-photon coupling in a one-dimensional quantum dot chain: Rabi waves and Rabi wave packets. Phys. Rev. B 2010, 81, 085115. [CrossRef]

42. Gligorić, G.; Maluckov, A.; Hadžievski, L.; Slepyan, G.Ya.; Malomed, B.A. Discrete solitons in an array of quantum dots. Phys. Rev. B 2013, 88, 155329. [CrossRef]

43. Chen, P.-Y.; Argyropoulos, C.; Alù, A. Enhanced nonlinearities using plasmonic nanoantennas. Nanophotonics 2012, 1, 221-233. [CrossRef]

44. Nielsen, M.A.; Chuang, I.L. Quantum Computation and Quantum Information; Cambridge University Press: Cambridge, UK, 2000.

45. Childs, A.M.; Gosset, D.; Webb, Z. Universal computation by multiparticle quantum walk. Science 2013, 339, 791-794. [CrossRef] [PubMed]

46. Cronin, A.D.; Schmiedmayer, J.; Pritchard, D.E. Optics and interferometry with atoms and molecules. Rev. Mod. Phys. 2009, 81, 1051. [CrossRef]

47. Mogilevtsev, D.; Slepyan, G.Y.; Garusov, E.; Kilin, S.Y.; Korolkova, N. Quantum tight-binding chains with dissipative coupling. New J. Phys. 2015, 17, 043065. [CrossRef]

48. Mogilevtsev, D.; Slepyan, G.Y. Diffusive lossless energy and coherence transfer by noisy coupling. Phys. Rev. A 2016, 94, 012116. [CrossRef] 
49. Mukherjee, S.; Mogilevtsev, D.; Slepyan, G.Y.; Doherty, T.H.; Thomson, R.R.; Korolkova, N. Dissipatively coupled waveguide networks for coherent diffusive photonics. Nat. Commun. 2018, 8, 1909. [CrossRef] [PubMed]

50. Luo, C.W.; Reimann, K.; Woerner, M.; Elsaesser, T.; Hey, R.; Ploog, K.H. Phase-Resolved Nonlinear Response of a Two-Dimensional Electron Gas under Femtosecond Intersubband Excitation. Phys. Rev. Lett. 2004, 92, 047402. [CrossRef] [PubMed]

51. Levie, I.; Kastner, R.; Slepyan, G. Rabi-Bloch oscillations in spatially distributed systems: Temporal dynamics and frequency spectra. Phys. Rev. A 2017, 96, 043854. [CrossRef]

52. Levie, I.; Slepyan, G. The New Concept of Nano-Device Spectroscopy Based on Rabi-Bloch Oscillations for THz-Frequency Range. Appl. Sci. 2017, 7, 721. [CrossRef]

53. Weißl, T.; Rastelli, G.; Matei, I.; Pop, I.M.; Buisson, O.; Hekking, F.W.J.; Guichard, W. Bloch band dynamics of a Josephson junction in an inductive environment. Phys. Rev. B 2015, 91, 014507. [CrossRef]

54. Nataf, P.; Ciuti, C. Vacuum Degeneracy of a Circuit QED System in the Ultrastrong Coupling Regime. Phys. Rev. Lett. 2010, 104, 023601. [CrossRef] [PubMed]

55. Guichard, W.; Hekking, F.W.J. Phase-charge duality in Josephson junction circuits: Role of inertia and effect of microwave irradiation. Phys. Rev. B 2010, 81, 064508. [CrossRef]

56. Manucharyan, V.E.; Masluk, N.A.; Kamal, A.; Koch, J.; Glazman, L.I.; Devoret, M.H. Evidence for coherent quantum phase slips across a Josephson junction array. Phys. Rev. B 2012, 85, 024521. [CrossRef]

57. Manfra, M.J. Molecular Beam Epitaxy of Ultra-High-Quality AlGaAs/GaAs Heterostructures: Enabling Physics in Low-Dimensional Electronic Systems. Annu. Rev. Condens. Matter Phys. 2014, 5, 347-373. [CrossRef]

58. Folsch, S.; Martinez-Blanco, J.; Yang, J.; Kanisawa, K.; Erwin, S.C. Quantum dots with single-atom precision. Nat. Nanotechnol. 2014, 9, 505. [CrossRef] [PubMed]

59. Heiss, M.; Fontana, Y.; Gustafsson, A.; Wüst, G.; Magen, C.; O’Regan, D.D.; Luo, J.W.; Ketterer, B.; Conesa-Boj, S.; Kuhlmann, A.V.; et al. Self-assembled quantum dots in a nanowire system for quantum photonics. Nat. Mater. 2013, 12, 439. [CrossRef] [PubMed]

60. Allen, L.; Eberly, J.H. Optical Resonance and Two-Level Atoms; Dover: New York, NY, USA, 1975.

61. Loudon, R. The Quantum Theory of Light; Clarendon: Oxford, UK, 1983.

62. Wubs, M.; Suttorp, L.G.; Lagendijk, A. Multiple-scattering approach to interatomic interactions and superradiance in inhomogeneous dielectrics. Phys. Rev. A 2004, 70, 053823. [CrossRef]

63. Sorensen, M.W.; Sorensen, A.S. Three-dimensional theory for light-matter interaction. Phys. Rev. A 2008, 77, 013826. [CrossRef]

64. Landau, L.D.; Lifshitz, E.M. Statistical Physics, Part 2, Course of Theoretical Physics; Pergamon Press: New York, NY, USA, 1980.

65. Todorov, Y.; Sirtori, C. Few-Electron Ultrastrong Light-Matter Coupling in a Quantum LC Circuit. Phys. Rev. X 2014, 4, 041031.

66. Vukics, A.; Grießer, T.; Domokos, P. Fundamental limitation of ultrastrong coupling between light and atoms. Phys. Rev. A 2015, 92, 043835. [CrossRef]

67. Soriano, A.; Navarro, E.A.; Porti, J.A.; Such, V. Analysis of the finite difference time domain technique to solve the Schrodinger equation for quantum devices. J. Appl. Phys. 2004, 95, 8011-8018. [CrossRef]

68. Sokolov, V.N.; Zhou, L.; Iafrate, G.J.; Krieger, J.B. Spontaneous emission of Bloch oscillation radiation from a single energy band. Phys. Rev. B 2006, 73, 205304. [CrossRef]

69. Yu, P.P.; Cardona, M. Fundamentals of Semiconductors: Physics and Material Properties; Springer: Berlin, Germany, 2001.

70. Landau, L.D.; Lifshitz, E.M. Quantum Mechanics, Course of Theoretical Physics; Pergamon Press: New York, NY, USA, 1965.

71. Keller, O. Quantum Theory of Near-Field Electrodynamics; Springer: Berlin, Germany, 2011.

(C) 2018 by the authors. Licensee MDPI, Basel, Switzerland. This article is an open access article distributed under the terms and conditions of the Creative Commons Attribution (CC BY) license (http:/ / creativecommons.org/licenses/by/4.0/). 